NTZ 20/97

July 1997

\title{
Complete control of gauge parameter dependence in the Abelian Higgs model
}

\author{
Rainer Häußling and Stephan Kappel \\ Institut für Theoretische Physik und \\ Naturwissenschaftlich-Theoretisches Zentrum, Universität Leipzig \\ Augustusplatz 10/11, D-04109 Leipzig, Germany
}

\begin{abstract}
We examine the dependence on all gauge parameters in the example of the Abelian Higgs model by applying a general algebraic method which roots in an extension of the usual Slavnov-Taylor identity. This method automatically yields all information about the gauge parameter dependence of Green functions and therefore especially allows to control the range of "good" normalization conditions. In this context we show that the physical on-shell normalization conditions are in complete agreement with the restrictions dictated by the enlarged Slavnov-Taylor identity and that the coupling can be fixed in an easily handleable way on the Ward identity of local gauge invariance. As an application of the general method we also study the Callan-Symanzik equation and the renormalization group equation of the Abelian Higgs model.
\end{abstract}




\section{Introduction}

The need to fix the gauge when quantizing a gauge theory perturbatively introduces a set of arbitrary gauge parameters into the action. Therefore one unavoidably has the task to control the dependence of the theory on these gauge parameters. Especially, it has to be proven that physical quantities indeed are gauge parameter independent. For instance, the gauge parameter independence of the S-matrix, already suggested in [1], was proven in [2] for gauge theories that do not contain any massless particles due to a complete spontaneous breakdown of symmetry. This proof, however, relies on a special set of on-shell normalization conditions and also makes use of a rather complicated technical tool, namely the Wilson operator product expansion. On the other hand, looking at pure gauge theories with massless gauge bosons, where the S-matrix does not exist, the gauge parameter independence of the $\beta$-functions has been shown. This, however, solely has been achieved by explicitly refering to an invariant renormalization scheme [3].

In the standard model of electroweak interactions the prerequisites needed for the proofs of the examples mentioned above are not fulfilled due to the masslessness of the photon and parity violation in the fermion sector. Hence the state of the art concerning the control of gauge parameter dependence is quite unsatisfactory and the necessity for having at hand a general (i.e. model- and scheme-independent) and easily manageable tool arises. Such a tool is given by the algebraic method first proposed in [4] which also allows for the control of gauge parameter dependence of single Green functions. As a preparatory step for similar investigations in the standard model this general method has been applied to the Abelian Higgs model in [5]. But in [5] attention was restricted to the dependence of the theory on one gauge parameter only. Among other things results proven in [6] by explicitly using an invariant scheme and special properties of the model could be reproduced in a model- and scheme-independent way. The present paper, now, enlarges the considerations of [5] to the full control of gauge parameter dependence (i.e. the control of the dependence of the theory on all gauge parameters) and hence completes the treatment of [5] in this sense. Again in view of the application of the algebraic method to the rather complicated standard model, this model containing quite a lot of gauge parameters, it seems to be instructive and in fact necessary to completely work out this method, applied in its full extent, in the simpler case of the Abelian Higgs model as a preliminary. The necessary prerequisites for an analogous discussion of gauge parameter dependence in the standard model are in the meanwhile available due to [7].

The algebraic method essentially roots in a certain extension of the ordinary BRS transformations: All the gauge parameters of the model now are allowed to transform under BRS into Grassmann variables. It then follows that constructing the Green functions in 
accordance with this enlarged BRS invariance also automatically yields all information about the gauge parameter dependence of the original Green functions, some of which are also used in the normalization conditions. Because these normalization conditions have to be chosen in agreement with the gauge parameter dependence of the theory (in order not to ruin, for instance, the gauge parameter independence of the S-matrix) we hence have at hand a powerful tool for controlling the range of allowed normalization conditions. In this context it turns out that the conditions of [2] and [3] just build special sets of adequate normalization conditions (c.f. section 5, [4, 8 ).

The structure of the paper will be as follows: In a first part (including sections 2-7) we enlarge the results obtained in [5] to the case when all gauge parameters undergo BRS transformations. This first part therefore parallels the discussion of [5] thereby putting emphasis on the modifications arising in the general, present, case. Whenever the treatment is completely analogous to the one in [5] we will skip calculational details and refer to [5], but nevertheless the present paper is fully self-contained as far as the line of argument is concerned: We start with a short recapitulation of the Abelian Higgs model (section 2) and the method of BRS transforming gauge parameters (section 3). In section 4 we will look for the solution of the classical approximation. This solution also leads to restrictions for the gauge parameter dependence of some of the free parameters of the model. Section 5 deals with the extension of these restrictions to higher orders of the perturbative expansion and shows the compatibility of the extended restrictions with physical on-shell normalization conditions. In sections 6 and 7 we prove global and local Ward identities. Section 7 also contains the discussion of an alternative and more elegant possibility for fixing the coupling.

In order to illustrate how far one can get with algebraic considerations alone and also to complete the algebraically abstract treatment we construct in a second part (sections 8, 9) parametric differential equations, namely the Callan-Symanzik equation and the renormalization group equation of the Abelian Higgs model. In this context we also discuss the dependence of the theory on the ghost mass.

Section 10 summarizes the results.

\section{The Abelian Higgs model}

We start with a short presentation of the Abelian Higgs model, thereby emphasizing some aspects which will become relevant in the following. The model consists of a doublet of scalar fields $\underline{\varphi}=\left(\varphi_{1}, \varphi_{2}\right)$ and a gauge field $A_{\mu}$ with an interaction, that breaks $U(1)$ 
gauge invariance spontaneously. In conventional normalization it can be described by the classical action

$$
\Gamma_{i n v}=\int\left\{-\frac{1}{4} F_{\mu \nu} F^{\mu \nu}+\frac{1}{2}\left(D_{\mu} \underline{\varphi}\right)\left(D^{\mu} \underline{\varphi}\right)-\frac{1}{8} \frac{m_{H}^{2}}{m^{2}} e^{2}\left(\varphi_{1}^{2}+2 \frac{m}{e} \varphi_{1}+\varphi_{2}^{2}\right)^{2}\right\}
$$

with:

$$
F_{\mu \nu} \equiv \partial_{\mu} A_{\nu}-\partial_{\nu} A_{\mu} \quad, \quad D_{\mu} \varphi_{1} \equiv \partial_{\mu} \varphi_{1}+e A_{\mu} \varphi_{2} \quad, \quad D_{\mu} \varphi_{2} \equiv \partial_{\mu} \varphi_{2}-e A_{\mu}\left(\varphi_{1}+\frac{m}{e}\right)
$$

$\Gamma_{i n v}$ respects $U(1)$ symmetry and the discrete symmetry of charge conjugation, i.e. it is invariant under the $U(1)$ transformations

$$
\delta_{\omega} A_{\mu}=\partial_{\mu} \omega, \delta_{\omega} \varphi_{1}=-e \omega \varphi_{2} \quad, \quad \delta_{\omega} \varphi_{2}=e \omega\left(\varphi_{1}+\frac{m}{e}\right)
$$

and under charge conjugation

$$
C A_{\mu}=-A_{\mu}, C \varphi_{1}=\varphi_{1}, C \varphi_{2}=-\varphi_{2}
$$

The shift $\frac{m}{e}$ of the field $\varphi_{1}$ produces the mass $m$ for the vector field $A_{\mu}$ and $\varphi_{1}$ is the physical Higgs field with mass $m_{H}$, whereas $\varphi_{2}$ takes the role of the would-be Goldstone boson eaten up by $A_{\mu}$.

In order to quantize the model the gauge has to be fixed. To this end we introduce an additional field, namely the auxiliary field $B$, with $\delta_{\omega} B=0$ and add a gauge fixing term

$$
\Gamma_{g . f .}=\int\left\{\frac{1}{2} \xi B^{2}+B\left(\partial A+\xi_{A} m \varphi_{2}\right)\right\} .
$$

$\xi$ and $\xi_{A}$ denote the gauge parameters, and the t'Hooft gauge fixing term $\int B \xi_{A} m \varphi_{2}$ is necessary in order to avoid a non-integrable infrared singularity in the $\left\langle\varphi_{2} \varphi_{2}\right\rangle$ propagator. Of course, this gauge fixing term violates both the local and global gauge invariance non-trivially:

$$
\delta_{\omega} \Gamma_{g . f .}=\int\left\{\omega \square B+e \omega B \xi_{A} m\left(\varphi_{1}+\frac{m}{e}\right)\right\}
$$

To retain a symmetry one has to further enlarge the model by introducing the FaddeevPopov $(\phi \pi)$ fields $c, \bar{c}$ and to extend local gauge transformations to BRS transformations:

$$
\begin{aligned}
s A_{\mu} & =\partial_{\mu} c, & s c & =0 \\
s \varphi_{1} & =-e c \varphi_{2}, & s \varphi_{2} & =e c\left(\varphi_{1}+\frac{m}{e}\right) \\
s \bar{c} & =B, & s B & =0
\end{aligned}
$$

Adding the $\phi \pi$-action,

$$
\Gamma_{\phi \pi}=\int\left\{-\bar{c} \square c-e \bar{c} \xi_{A} m\left(\varphi_{1}+\frac{m}{e}\right) c\right\}
$$


the BRS variation of $\Gamma_{\phi \pi}$ exactly cancels the BRS variation of $\Gamma_{g . f}$, and $\Gamma_{i n v}+\Gamma_{g . f .}+\Gamma_{\phi \pi}$ is BRS symmetric. The BRS symmetry is a powerful technical tool which is essential for the proof of renormalizability and unitarity of the S-matrix. It also defines the model in question in an implicit way (see below).

Finally, we have to care about the non-linear BRS transformations $s \varphi_{i}$ which are not well-defined in higher orders of perturbation theory due to their non-linearity. In order to circumvent this difficulty we couple these BRS variations to external fields $Y_{i}$ with $s Y_{i}=0$ and add an external field part:

$$
\Gamma_{\text {e.f. }}=\int\left\{Y_{1}\left(s \varphi_{1}\right)+Y_{2}\left(s \varphi_{2}\right)\right\}
$$

The complete BRS invariant classical action is now given by:

$$
\Gamma_{c l}=\Gamma_{i n v}+\Gamma_{g . f .}+\Gamma_{\phi \pi}+\Gamma_{e . f}
$$

A further complication arises when looking at rigid and local gauge invariance in terms of Ward identities: In [9] it has been shown that a proper formulation of rigid and local gauge symmetry (to all orders) is achieved by complementing the gauge fixing by an doublet of external scalar fields $\underline{\hat{\varphi}}=\left(\hat{\varphi}_{1}, \hat{\varphi}_{2}\right)$ :

$$
\Gamma_{g . f .}=\int\left\{\frac{1}{2} \xi B^{2}+B \partial A-e B\left(\left(\hat{\varphi}_{1}-\xi_{A} \frac{m}{e}\right) \varphi_{2}-\hat{\varphi}_{2}\left(\varphi_{1}-\hat{\xi}_{A} \frac{m}{e}\right)\right)\right\}
$$

The doublet $\underline{\hat{\varphi}}$ transforms under $U(1)$ according to:

$$
\delta_{\omega} \hat{\varphi}_{1}=-e \omega \hat{\varphi}_{2}, \delta_{\omega} \hat{\varphi}_{2}=e \omega\left(\hat{\varphi}_{1}-\xi_{A} \frac{m}{e}\right)
$$

Then $\Gamma_{\text {g.f. }}$ (2.11) is invariant under the global $U(1)$ transformations (2.3), (2.12) if we choose $\hat{\xi}_{A}=-1$, and the original gauge fixing (2.5) is recovered for $\hat{\varphi}_{i}=0$.

The external fields $\hat{\varphi}_{i}$ are transformed under BRS into another doublet of external fields $\underline{q}=\left(q_{1}, q_{2}\right)$ :

$$
s \hat{\varphi}_{i}=q_{i}, s q_{i}=0, i=1,2
$$

The BRS invariance of the theory is expressed by the Slavnov-Taylor (ST) identity

$$
\mathcal{S}(\Gamma) \equiv \int\left\{\partial_{\mu} c \frac{\delta \Gamma}{\delta A_{\mu}}+B \frac{\delta \Gamma}{\delta \bar{c}}+\frac{\delta \Gamma}{\delta \underline{Y}} \frac{\delta \Gamma}{\delta \underline{\varphi}}+\underline{q} \frac{\delta \Gamma}{\delta \underline{\hat{\varphi}}}\right\}=0
$$

At the classical level $\Gamma$ is just the classical action $\Gamma_{c l}$, whereas at the quantum level $\Gamma$ denotes the vertex functional $\Gamma=\Gamma_{c l}+O(\hbar)$. It can be proven that (2.14) together with appropriate normalization conditions, invariance under charge conjugation and the gauge condition (2.11) uniquely defines the model to all orders of perturbation theory. This is in contrast to the standard model where in addition to the ST identity also rigid 


\begin{tabular}{l|c|c|c|c|c|c|c|c|c|c} 
fields & $A_{\mu}$ & $B$ & $\tilde{\varphi}_{1}$ & $\tilde{\varphi}_{2}$ & $c$ & $\bar{c}$ & $Y_{1}$ & $Y_{2}$ & $q_{1}$ & $q_{2}$ \\
\hline $\operatorname{dim}$ & 1 & 2 & 1 & 1 & 0 & 2 & 3 & 3 & 1 & 1 \\
\hline charge conj. & - & - & + & - & - & - & + & - & + & - \\
\hline$Q_{\phi \pi}$ & 0 & 0 & 0 & 0 & +1 & -1 & -1 & -1 & +1 & +1
\end{tabular}

Table 1: Quantum numbers of the fields $\left(\tilde{\varphi}_{i}=\varphi_{i}, \hat{\varphi}_{i}\right)$

invariance, a local Ward identity and some consistency relations are needed for a full algebraic characterization of the model [7].

In a first step one has to look for the most general, field polynomial (i.e. classical) solution $\Gamma_{c l}^{g e n}$ of the ST identity (2.14) and the gauge condition (2.11) which is invariant under charge conjugation, and to prove that it coincides with $\Gamma_{c l}$ after the application of appropriate normalization conditions. This procedure also yields information about all the free parameters of the theory. The most general solution was calculated in [9] and is presented in appendix A. The free parameters in $\Gamma_{c l}^{g e n}$ are the usual field and coupling renormalizations $z_{1}, z_{2}, z_{A}, z_{m}, z_{m_{H}}, z_{e}$,

$$
\begin{gathered}
\varphi_{i} \longrightarrow \sqrt{z_{i}}\left(\varphi_{i}-x_{i} \hat{\varphi}_{i}\right), A_{\mu} \longrightarrow \sqrt{z_{A}} A_{\mu} \\
m \longrightarrow \sqrt{z_{m}} m, m_{H} \longrightarrow \sqrt{z_{m_{H}}} m_{H}, e \longrightarrow z_{e} e,
\end{gathered}
$$

as well as the gauge parameters $\xi, \xi_{A}$, the parameter $\mu$ (see appendix A), $\hat{\xi}_{A}$ (which is prescribed by the global Ward identity, see (6.3)) and the two parameters $x_{1}, x_{2}$, which appear in the combination

$$
\bar{\varphi}_{i}=\varphi_{i}-x_{i} \hat{\varphi}_{i}
$$

that replaces $\varphi_{i}$ in $\Gamma_{i n v}$.

These parameters have to be fixed by normalization conditions in each order of the perturbative expansion. In the following we will choose (for reasons which will become clear later on) physical on-shell normalization conditions:

$$
\begin{aligned}
& \operatorname{Re} \Gamma_{\varphi_{1} \varphi_{1}}\left(p^{2}=m_{H}^{2}\right)=0 \text { fixes } z_{m_{H}} \\
& \Gamma^{T}\left(p^{2}=m^{2}\right)=0 \text { fixes } z_{m} \\
& \Gamma_{c \bar{c}}\left(p^{2}=m_{\text {ghost }}^{2}\right)=0, m_{\text {ghost }}^{2}=\xi_{A} m^{2} \quad \text { fixes } \xi_{A} \\
& \partial_{p^{2}} \Gamma^{T}\left(p^{2}=m^{2}\right)=1 \text { fixes } z_{A} \\
& \text { Re } \partial_{p^{2}} \Gamma_{\varphi_{1} \varphi_{1}}\left(p^{2}=m_{H}^{2}\right)=1 \text { fixes } z_{1} \\
& \partial_{p^{2}} \Gamma_{\varphi_{2} \varphi_{2}}\left(p^{2}=\kappa^{2}\right)=1 \text { fixes } z_{2}
\end{aligned}
$$




$$
\begin{gathered}
\Gamma_{Y_{1} q_{1}}\left(p^{2}=\kappa^{2}\right)=x_{1}^{(0)} \text { fixes } x_{1} \\
\Gamma_{Y_{2} q_{2}}\left(p^{2}=\kappa^{2}\right)=x_{2}^{(0)} \text { fixes } x_{2} \\
\Gamma_{\varphi_{1}}=0 \text { fixes } \mu
\end{gathered}
$$

In (2.17), (2.18) the transversal part of the vector 2-point function is given by:

$$
\Gamma_{A^{\mu} A^{\nu}}(p,-p) \equiv \Gamma_{\mu \nu}(p,-p)=\left(\eta_{\mu \nu}-\frac{p_{\mu} p_{\nu}}{p^{2}}\right) \Gamma^{T}\left(p^{2}\right)+\frac{p_{\mu} p_{\nu}}{p^{2}} \Gamma^{L}\left(p^{2}\right)
$$

It remains to give a normalization condition for the coupling $e$. Preliminary (see sections 5 and 7 ), we fix the coupling on the 3 -point vertex function $\Gamma_{A_{\mu} \varphi_{1} \varphi_{2}}$ at a normalization momentum $p_{\text {norm }}$ :

$$
\left.\partial_{p_{1}^{\nu}} \Gamma_{A_{\mu} \varphi_{1} \varphi_{2}}\left(-p_{1}-p_{2}, p_{1}, p_{2}\right)\right|_{\left\{p_{i}\right\}=p_{\text {norm }}}=-i e \eta^{\mu \nu} \quad \text { fixes } \quad z_{e}
$$

It is easily checked that these normalization conditions when applied to the tree approximation $\Gamma_{c l}^{g e n}$ (see appendix A) exactly yield $\Gamma_{c l}$ (2.10) (if we set $x_{i}=0$ ).

\section{Algebraic control of gauge parameter dependence}

We now want to turn to the proper subject of the present paper, namely the control of gauge parameter dependence. To this end we first observe that at the level of the classical action $\Gamma_{c l}(2.10)$ the dependence on the two gauge parameters $\xi$ and $\xi_{A}$ is given by two BRS variations,

$$
\begin{gathered}
\partial_{\xi} \Gamma_{c l}=\frac{1}{2} \int B^{2}=\frac{1}{2} s \int \bar{c} B \quad \text { and } \\
\partial_{\xi_{A}} \Gamma_{c l}=m \int\left\{B \varphi_{2}-e \bar{c}\left(\varphi_{1}+\frac{m}{e}\right) c\right\}=m s \int \bar{c} \varphi_{2}
\end{gathered}
$$

respectively. Therefore the right hand sides of (3.1) vanish between physical states and physical quantities (like the S-matrix) are $\xi$ - and $\xi_{A}$-independent in the tree approximation.

The question now arises whether - and if yes, how - this statement can be extended to higher orders. In the affirmative case we furthermore would like to use a construction which is easily manageable and which does not rely on the specific model and/or a specific renormalization scheme. Of course, if the model in question permits a gauge-invariant regularization, such a general approach does not seem to be necessary at first sight. But because many models lack this property it is nevertheless desirable to have in hand such

a model- and scheme-independent procedure for controlling gauge parameter dependence 
and to see how it works. In addition, it will turn out that some quite general results are only (or at least much more easily) accessible with the proposed method.

For this purpose let us therefore allow the gauge parameters $\xi$ and $\xi_{A}$ to transform under BRS into Grassmann variables $\chi$ and $\chi_{A}$, respectively, with $\phi \pi$-charge +1 [四]:

$$
s \xi=\chi \quad, \quad s \xi_{A}=\chi_{A} \quad, \quad s \chi=0=s \chi_{A}
$$

Hence, the ST identity modifies into:

$$
\mathcal{S}(\Gamma)+\chi \partial_{\xi} \Gamma+\chi_{A} \partial_{\xi_{A}} \Gamma=0
$$

Differentiation of (3.3) with respect to $\chi$ or $\chi_{A}$ and evaluating the results at $\chi=0=\chi_{A}$ leads to

$$
\begin{aligned}
&-\left.s_{\Gamma}^{\chi=0=\chi_{A}}\left(\partial_{\chi} \Gamma\right)\right|_{\chi=0=\chi_{A}}+\left.\partial_{\xi} \Gamma\right|_{\chi=0=\chi_{A}}=0, \\
&-\left.s_{\Gamma}^{\chi=0=\chi_{A}}\left(\partial_{\chi_{A}} \Gamma\right)\right|_{\chi=0=\chi_{A}}+\left.\partial_{\xi_{A}} \Gamma\right|_{\chi=0=\chi_{A}}=0,
\end{aligned}
$$

where in the model under investigation $s_{\Gamma}$ is given by:

$$
s_{\Gamma}=\int\left\{\partial c \frac{\delta}{\delta A}+B \frac{\delta}{\delta \bar{c}}+\frac{\delta \Gamma}{\delta \underline{Y}} \frac{\delta}{\delta \underline{\varphi}}+\frac{\delta \Gamma}{\delta \underline{\varphi}} \frac{\delta}{\delta \underline{Y}}+\underline{q} \frac{\delta}{\delta \underline{\varphi}}\right\}+\chi \partial_{\xi}+\chi_{A} \partial_{\xi_{A}}
$$

$s_{\Gamma}$ being - roughly speaking - the functional generalization of $s$, eq. (3.4) is nothing else but the functional analog of (3.1) which we were looking for and which can be easily controlled in higher orders. Therefore proving (3.3) to all orders of perturbation theory automatically yields all information about gauge parameter dependence of the 1-PI Green functions in an algebraic way.

\section{Slavnov-Taylor identity for $\chi \neq 0, \chi_{A} \neq 0$}

In accordance with the observations of the proceeding section gauge parameter dependence is completely governed by the $\chi$ - and $\chi_{A}$-enlarged ST identity

$$
\mathcal{S}(\Gamma) \equiv\left\{\partial_{\mu} c \frac{\delta \Gamma}{\delta A_{\mu}}+B \frac{\delta \Gamma}{\delta \bar{c}}+\frac{\delta \Gamma}{\delta \underline{Y}} \frac{\delta \Gamma}{\delta \underline{\varphi}}+\underline{q} \frac{\delta \Gamma}{\delta \underline{\varphi}}\right\}+\chi \partial_{\xi} \Gamma+\chi_{A} \partial_{\xi_{A}} \Gamma=0
$$

First we have to look for the general classical solution $\Gamma=\Gamma_{c l}^{g e n}$ of (4.1) in order to control the free parameters of the theory and to learn something about their gauge parameter

\footnotetext{
${ }^{1}$ From here on the symbol $\mathcal{S}$ collectively denotes all the differential operators on the r.h.s. of (3.3)
} 
dependence eventually. Because the ST identity does not prescribe the gauge fixing terms we can also postulate the gauge condition (2.11)

$$
\left.\frac{\delta \Gamma}{\delta B}\right|_{\chi=0=\chi_{A}}=\xi B+\partial A-e\left[\left(\hat{\varphi}_{1}-\xi_{A} \frac{m}{e}\right) \varphi_{2}-\hat{\varphi}_{2}\left(\varphi_{1}-\hat{\xi}_{A} \frac{m}{e}\right)\right]
$$

to hold for the solution $\Gamma$ of (4.1). The gauge condition (4.2) is linear in propagating fields and hence it can be integrated in this form to all orders of perturbation theory.

Using the fact that $\chi$ and $\chi_{A}$ are Grassmann variables, $\Gamma$ can be split into four parts in the tree approximation:

$$
\Gamma=\hat{\Gamma}+\chi Q+\chi_{A} Q_{A}+\chi \chi_{A} Q_{\chi \chi_{A}}
$$

Inserting (4.3) into the ST identity (4.1) and again making use of $\chi^{2}=0=\chi_{A}^{2}$ one immediately finds that at the classical level (4.1) is equivalent to the following four equations:

$$
\begin{array}{lll}
\chi^{0}, \chi_{A}^{0} & : & \int\left\{\partial_{\mu} c \frac{\delta \hat{\Gamma}}{\delta A_{\mu}}+B \frac{\delta \hat{\Gamma}}{\delta \bar{c}}+\frac{\delta \hat{\Gamma}}{\delta \underline{Y}} \frac{\delta \hat{\Gamma}}{\delta \underline{\varphi}}+\underline{q} \frac{\delta \hat{\Gamma}}{\delta \underline{\hat{\varphi}}}\right\}=0 \\
\chi^{1}, \chi_{A}^{0}: & \partial_{\xi} \hat{\Gamma}=s_{\hat{\Gamma}}^{\chi=0=\chi_{A}} Q \\
\chi^{0}, \chi_{A}^{1}: & \partial_{\xi_{A}} \hat{\Gamma}=s_{\hat{\Gamma}}^{\chi=0=\chi_{A}} Q_{A} \\
\chi^{1}, \chi_{A}^{1} & : & \int\left\{\frac{\delta Q}{\delta \underline{Y}} \frac{\delta Q_{A}}{\delta \underline{\varphi}}-\frac{\delta Q_{A}}{\delta \underline{Y}} \frac{\delta Q}{\delta \underline{\varphi}}\right\}-\partial_{\xi} Q_{A}+\partial_{\xi_{A}} Q=s_{\hat{\Gamma}}^{\chi=0=\chi_{A}} Q_{\chi_{\chi_{A}}}
\end{array}
$$

$s_{\hat{\Gamma}}$ is given by $(3.5)$ (with $\hat{\Gamma}$ replacing $\Gamma$ ).

The first of these equations is nothing else but the (ordinary) ST identity for $\chi=0=\chi_{A}$ which has been studied in [9] and the general solution of which - needed for the calculation of $Q$ and $Q_{A}$ - is presented in appendix A.

Furthermore, (4.3) implies that $Q, Q_{A}$ and $Q_{\chi_{\chi_{A}}}$ have dimension less than or equal to four and are even under charge conjugation and that $Q$ as well as $Q_{A}$ carry $\phi \pi$-charge -1 whereas $Q_{\chi \chi_{A}}$ has $\phi \pi$-charge -2 . According to the table of quantum numbers the most general ansatz for $Q$ is hence given by:

$$
\begin{aligned}
Q=\int & \left\{d_{1} Y_{1} \varphi_{1}+\hat{d}_{1} Y_{1} \hat{\varphi}_{1}+d Y_{1}+d_{2} Y_{2} \varphi_{2}+\hat{d}_{2} Y_{2} \hat{\varphi}_{2}\right. \\
& f \bar{c} \varphi_{2}+\hat{f} \bar{c} \hat{\varphi}_{2}+\tilde{f} \bar{c} B+f_{A} \bar{c} \partial_{\mu} A^{\mu} \\
& \left.h_{1} \bar{c} \varphi_{1} \varphi_{2}+h_{2} \bar{c} \hat{\varphi}_{1} \varphi_{2}+h_{3} \bar{c} \varphi_{1} \hat{\varphi}_{2}+h_{4} \bar{c} \hat{\varphi}_{1} \hat{\varphi}_{2}\right\}
\end{aligned}
$$

For $Q_{A}$ the same ansatz holds true but with a new set of 13 parameters $d_{1}^{A}, \hat{d}_{1}^{A}, \ldots, h_{4}^{A}$ instead of $d_{1}, \hat{d}_{1}, \ldots, h_{4}$. Due to the quantum numbers of $Q_{\chi \chi_{A}}$ there are no terms contributing to $Q_{\chi \chi_{A}}$ :

$$
Q_{\chi \chi_{A}} \equiv 0
$$


Putting (4.8) and the analogous expression for $Q_{A}$ into (4.5), (4.6), respectively, yields after a straightforward calculation the determination of the 26 parameters $d_{1}, \ldots, h_{4}, d_{1}^{A}, \ldots$, $h_{4}^{A}$; we finally find $\left(Q_{(A)}=Q, Q_{A}\right)$ :

$$
Q_{(A)}=Q_{e . f .(A)}+Q_{\phi \pi, 1(A)}+Q_{\phi \pi, 2(A)}
$$

with $\left(x_{1}^{(0)}=x_{2}^{(0)} \equiv x(\right.$ see $(6.3))$ and $\left.\bar{\varphi}_{i}=\varphi_{i}-x \hat{\varphi}_{i}\right)$ :

$$
\begin{aligned}
Q_{e . f .(A)}= & \int\left\{\frac{1}{4}\left(\partial_{\xi_{(A)}} \ln z_{1}+\partial_{\xi_{(A)}} \ln z_{2}\right)\left(Y_{1} \bar{\varphi}_{1}+Y_{2} \bar{\varphi}_{2}\right)\right. \\
& \left.+\frac{1}{4}\left(\partial_{\xi_{(A)}} \ln z_{1}-\partial_{\xi_{(A)}} \ln z_{2}\right)\left(Y_{1} \bar{\varphi}_{1}-Y_{2} \bar{\varphi}_{2}\right)-\partial_{\xi_{(A)}} x\left(Y_{1} \hat{\varphi}_{1}+Y_{2} \hat{\varphi}_{2}\right)\right\} \\
Q_{\phi \pi, 1(A)}= & \int\left\{-\frac{1}{4} e \bar{c}\left(\partial_{\xi_{(A)}} \ln z_{1}+\partial_{\xi_{(A)}} \ln z_{2}\right)\left(\left(\bar{\varphi}_{1}+\frac{\sqrt{z_{m}}}{\sqrt{z_{1}} z_{e}} \frac{m}{e}\right) \hat{\varphi}_{2}-\bar{\varphi}_{2}\left(\hat{\varphi}_{1}-\xi_{A} \frac{m}{e}\right)\right)\right. \\
& \left.-\frac{1}{4} e \bar{c}\left(\partial_{\xi_{(A)}} \ln z_{1}-\partial_{\xi_{(A)}} \ln z_{2}\right)\left(\left(\bar{\varphi}_{1}+\frac{\sqrt{z_{m}}}{\sqrt{z_{1}} z_{e}} \frac{m}{e}\right) \hat{\varphi}_{2}+\bar{\varphi}_{2}\left(\hat{\varphi}_{1}-\xi_{A} \frac{m}{e}\right)\right)\right\} \\
Q_{\phi \pi, 2}= & \frac{1}{2} \int \bar{c} B \\
Q_{\phi \pi, 2 A}= & m \int \bar{c} \bar{\varphi}_{2}
\end{aligned}
$$

Please note that with (4.10) eq. (4.7) is fulfilled automatically.

Hence the coefficients in $Q$ and $Q_{A}$ are completely determined as functions of the parameters $z_{1}, z_{2}, z_{m}, z_{e}$ and $x$ which appear in the general solution of the ST identity for $\chi=0=\chi_{A}$. But the $\chi$ - and $\chi_{A}$-enlarged ST identity does not only fully fix $Q$ and $Q_{A}$; in addition (4.5), (4.6) force some of the free parameters to be both $\xi$ - and $\xi_{A}$-independent:

$$
\begin{gathered}
\partial_{\xi} z_{e}=0=\partial_{\xi_{A}} z_{e}, \partial_{\xi} z_{A}=0=\partial_{\xi_{A}} z_{A}, \\
\partial_{\xi} z_{m}=0=\partial_{\xi_{A}} z_{m} \quad, \quad \partial_{\xi} z_{m_{H}}=0=\partial_{\xi_{A}} z_{m_{H}} \quad, \quad \partial_{\xi} \mu^{2}=0=\partial_{\xi_{A}} \mu^{2}
\end{gathered}
$$

In contrast to this the wave function renormalizations $z_{1}, z_{2}$ and $x$ can be arbitrary functions of $\xi$ and $\xi_{A}$.

Two remarks are of some relevance at this point:

The (physical) normalization conditions given in section 2 trivially fulfil the constraints (4.15) in the tree approximation. In higher orders of perturbation theory, however, the constraints (4.15) will extend to restrictions of the $\xi$ - and $\xi_{A}$-dependence of some non-local Green functions (the subject of the next section) which are also used in the normalization conditions and the splitting of which into $\xi\left(\xi_{A}\right)$-dependent and $\xi\left(\xi_{A}\right)$-independent parts is much less transparent. Hence some care is needed in order not to introduce wrong gauge parameter dependence into the theory, i.e. it has to be proven explicitly that the normalization conditions chosen are in agreement with the restrictions (4.15) extended to 
higher orders.

The second remark concerns the t'Hooft gauge

$$
\xi_{A}=\xi
$$

which seems to be excluded in the present treatment because $\xi$ and $\xi_{A}$ are viewed as being independent gauge parameters. But with the following recipe it is nevertheless possible to make a transition from the general to the t'Hooft case:

- Set $\partial_{\xi_{A}}$ equal to zero in all places of occurence, this partial derivative having already

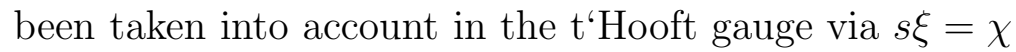

- Take then $\chi_{A}=\chi$

It is easily seen that this procedure leads to the correct results.

\section{Gauge parameter dependence of Green functions}

The next step would be the proof of the $\chi$ - and $\chi_{A}$-dependent ST identity (4.1) to all orders of perturbation theory. We will not present the detailed proof here but instead refer to [4] where it was shown that the proof of the enlarged ST identity $\left(\chi \neq 0, \chi_{A} \neq 0\right)$ can be reduced to the proof of the ordinary ST identity $\left(\chi=0=\chi_{A}\right)$ : The only possible obstruction to the validity of the ST identity would be the presence of anomalies which, however, are absent in the Abelian Higgs model. Hence we can acchieve

$$
\mathcal{S}(\Gamma)=0
$$

also in the case of BRS transforming gauge parameters $\xi$ and $\xi_{A}$, namely by an appropriate choice of counterterms. $\Gamma$ now denotes the generating functional of 1-PI Green functions. Accordingly the validity of (5.1) will be assumed throughout the following.

We now want to deal with the extensions of the constraints (4.15) to higher orders't. Because this discussion again parallels the analogous discussion of [5] for one BRS transforming gauge parameter we skip the details of the calculations here.

The fundamental starting point for all considerations that follow are the equations (3.4) which have to be differentiated with respect to suitable fields and finally evaluated for all fields equal to zero.

\footnotetext{
${ }^{2}$ In this context we will restrict ourselfes to the case of a stable Higgs particle, i.e. $m_{H}^{2}<4 m^{2}$.
} 
Let us start with a more technical point: the continuation of the $\xi$-and $\xi_{A}$-independence of $\mu^{2}$ to higher orders. To this end we differentiate (3.4) with respect to $\varphi_{1}$; in momentum space we get:

$$
\partial_{\chi_{(A)}} \Gamma_{Y_{1}}(0) \Gamma_{\varphi_{1} \varphi_{1}}(0)+\partial_{\chi_{(A)}} \Gamma_{Y_{1} \varphi_{1}}(0) \Gamma_{\varphi_{1}}(0)=-\partial_{\xi_{(A)}} \Gamma_{\varphi_{1}}(0)
$$

Using the normalization condition $\Gamma_{\varphi_{1}}=0$ (2.20), eq. (5.2) simplifies to

$$
\partial_{\chi_{(A)}} \Gamma_{Y_{1}}(0)\left(-m_{H}^{2}+\mathcal{O}(\hbar)\right)=0
$$

from which it follows that

$$
\partial_{\chi} \Gamma_{Y_{1}}=0 \text { and } \partial_{\chi_{A}} \Gamma_{Y_{1}}=0
$$

hold to all orders of the perturbative expansion.

We next come to the proof of the statement that the transversal part of the vector 2-point function is completely gauge parameter independent (Classically this statement is true due to $\partial_{\xi} z_{A}=0=\partial_{\xi_{A}} z_{A}$ and $\partial_{\xi} z_{m}=0=\partial_{\xi_{A}} z_{m}$.): Differentiation of (3.4) with respect to $A_{\mu}$ and $A_{\nu}$ leads to (thereby using (5.4)):

$$
\partial_{\chi_{(A)}} \Gamma_{Y_{2} A_{\mu}}(p,-p) \Gamma_{\varphi_{2} A_{\nu}}(p,-p)+(\mu \leftrightarrow \nu)=-\partial_{\xi_{(A)}} \Gamma_{A_{\mu} A_{\nu}}(p,-p)
$$

A simple argument using Lorentz invariance (see also [5]) shows that the left hand sides of (5.5) only contribute to the longitudinal part of $\partial_{\xi} \Gamma_{A_{\mu} A_{\nu}}$ and $\partial_{\xi_{A}} \Gamma_{A_{\mu} A_{\nu}}$, respectively. Therefore we get the desired result:

$$
\partial_{\xi} \Gamma_{A_{\mu} A_{\nu}}^{T}=0 \quad \text { and } \quad \partial_{\xi_{A}} \Gamma_{A_{\mu} A_{\nu}}^{T}=0
$$

(The transversal part of $\Gamma_{A_{\mu} A_{\nu}}$ is defined in (2.21).) Finally it is easy (but nevertheless necessary) to prove that the on-shell normalization conditions (2.17), (2.18) involving $\Gamma_{A_{\mu} A_{\nu}}^{T}$ are in agreement with the constraints (5.6).

Please also note that the restrictions found above for the transversal part of the vector 2-point function are only available in this simple way by controlling gauge parameter dependence algebraically.

In a quite analogous manner the constraint $\partial_{\xi} z_{m_{H}}=0=\partial_{\xi_{A}} z_{m_{H}}$ is extended to higher orders: This time we differentiate (3.4) twice with respect to $\varphi_{1}$ (and use again (5.4)):

$$
\partial_{\chi_{(A)}} \Gamma_{Y_{1} \varphi_{1}}\left(p^{2}\right) \Gamma_{\varphi_{1} \varphi_{1}}\left(p^{2}\right)=-\partial_{\xi_{(A)}} \Gamma_{\varphi_{1} \varphi_{1}}\left(p^{2}\right)
$$

Equation (5.7) completely governs the $\xi$ - and $\xi_{A}$-dependence of the Higgs self-energy. But due to the existence of non-trivial insertions of the vertices $\chi \bar{c} B$ and $\chi_{A} m \bar{c} \varphi_{2}$ into the 
vertex function $\Gamma_{Y_{1} \varphi_{1}}$ the l.h.s of (5.7) is not trivial at all, this being in contrast to the discussion of the transversal part of the vector 2-point function. Nevertheless, it is easily shown (order by order in perturbation theory) that the on-shell normalization condition (2.17) is in agreement with the constraint (5.7). (See [5] for a more detailed discussion.)

We want to conclude this section by making some remarks concerning the extension of the constraints $\partial_{\xi} z_{e}=0=\partial_{\xi_{A}} z_{e}$ to higher orders. Testing (3.4) with respect to $A_{\mu}, \varphi_{1}$ and $\varphi_{2}$ leads to:

$$
\begin{aligned}
\partial_{\chi_{(A)}} \Gamma_{Y_{1} \varphi_{1}}\left(p_{1}^{2}\right) \Gamma_{\varphi_{1} \varphi_{2} A_{\mu}}\left(p_{1}, p_{2}, p\right) & +\partial_{\chi_{(A)}} \Gamma_{Y_{2} \varphi_{2}}\left(p_{2}^{2}\right) \Gamma_{\varphi_{1} \varphi_{2} A_{\mu}}\left(p_{1}, p_{2}, p\right) \\
+\partial_{\chi_{(A)}} \Gamma_{Y_{2} \varphi_{1} A_{\mu}}\left(p_{2}, p_{1}, p\right) \Gamma_{\varphi_{2} \varphi_{2}}\left(p_{2}^{2}\right) & +\partial_{\chi_{(A)}} \Gamma_{Y_{1} \varphi_{2} A_{\mu}}\left(p_{1}, p_{2}, p\right) \Gamma_{\varphi_{1} \varphi_{1}}\left(p_{1}^{2}\right) \\
+\partial_{\chi_{(A)}} \Gamma_{Y_{2} \varphi_{1} \varphi_{2}}\left(p, p_{1}, p_{2}\right) \Gamma_{\varphi_{2} A_{\mu}}(-p, p) & =-\partial_{\xi_{(A)}} \Gamma_{\varphi_{1} \varphi_{2} A_{\mu}}\left(p_{1}, p_{2}, p\right)
\end{aligned}
$$

The left hand sides of (5.8) do not contain any free parameters once the residua of the Higgs and the would-be Goldstone are fixed by the normalization conditions (2.18). Hence

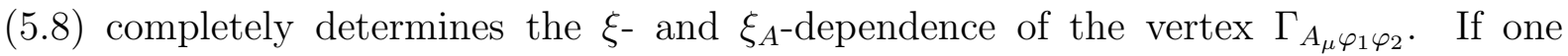
therefore insists in fixing the coupling directly with the help of $\Gamma_{A_{\mu} \varphi_{1} \varphi_{2}}$ (as it was done in (2.22) ) one has to introduce two reference points $\xi_{0}$ and $\xi_{A_{0}}$ in order to fix the $\xi$ - and $\xi_{A^{-}}$-independent part of $\Gamma_{A_{\mu} \varphi_{1} \varphi_{2}}$,

$$
\left.\partial_{p_{1}^{\nu}} \Gamma_{A_{\mu} \varphi_{1} \varphi_{2}}\left(-p_{1}-p_{2}, p_{1}, p_{2}\right)\right|_{\left\{p_{i}\right\}=p_{\text {norm }}, \xi_{=}=\xi_{0}, \xi_{A}=\xi_{A_{0}}}=-i e \eta^{\mu \nu}
$$

and to govern $\xi$ - and $\xi_{A}$-dependence via (5.8). Such a procedure, however, is not evident and easily manageable in explicit calculations at all. In section 7 we will see that in the Abelian Higgs model there is a much more elegant and practicable way of fixing the coupling, namely by making use of the local Ward identity.

\section{Rigid invariance}

In 99 it was proven that the $\chi^{-}$and $\chi_{A}$-independent part of the generating functional of 1-PI Green functions obeys a Ward identity of rigid symmetry to all orders of perturbation theory,

$$
\left.\hat{W}^{\text {gen }} \Gamma\right|_{\chi=0=\chi_{A}}=0 \text {, }
$$

where $\hat{W}^{\text {gen }}$ denotes the (deformed) Ward operator:

$$
\begin{aligned}
\hat{W}^{g e n} \equiv \int & \left\{-z^{-1} \varphi_{2} \frac{\delta}{\delta \varphi_{1}}+z\left(\varphi_{1}-\hat{\xi}_{A} \frac{m}{e}\right) \frac{\delta}{\delta \varphi_{2}}-z Y_{2} \frac{\delta}{\delta Y_{1}}+z^{-1} Y_{1} \frac{\delta}{\delta Y_{2}}\right. \\
& \left.-z^{-1} \hat{\varphi}_{2} \frac{\delta}{\delta \hat{\varphi}_{1}}+z\left(\hat{\varphi}_{1}-\xi_{A} \frac{m}{e}\right) \frac{\delta}{\delta \hat{\varphi}_{2}}-z^{-1} q_{2} \frac{\delta}{\delta q_{1}}+z q_{1} \frac{\delta}{\delta q_{2}}\right\}
\end{aligned}
$$


The appearance of a deformed Ward operator is due to the fact that physical on-shell normalization conditions (which are "good" normalization conditions, see section 5) have been used. In other words: The WI (6.1) does not prescribe the values of $z$ and $\xi_{A}$, instead these parameters are fixed uniquely by explicit normalization conditions, namely the normalization conditions imposed on the residua of the Higgs and Goldstone field (2.18) and the mass normalization of the ghosts and the Higgs (2.17).

Nevertheless, (6.1) restricts some other parameters at the classical level:

$$
x_{1}^{(0)}=x_{2}^{(0)} \equiv x \quad, \quad \hat{\xi}_{A}=-1+x \xi_{A}
$$

Now we are going to study the modifications of (6.1) when BRS transforming gauge parameters $\xi$ and $\xi_{A}$ are included. We will start with a more detailed investigation of the classical approximation, these considerations yielding a hint of what could be expected in higher orders, then we will outline the essential steps for the proof of the WI obtained classically to all orders of perturbation theory.

Acting with $\hat{W}^{\text {gen }}$ (6.2) on the general solution $\Gamma_{c l}^{g e n}$ (4.3) of the ST identity (4.1) yields (using $\hat{W}^{g e n} \hat{\Gamma}_{c l}^{g e n}=0$ ):

$$
\hat{W}^{g e n} \Gamma_{c l}^{g e n}=\hat{W}^{g e n}\left(\hat{\Gamma}_{c l}^{g e n}+\chi Q+\chi_{A} Q_{A}\right)=\chi \hat{W}^{g e n} Q+\chi_{A} \hat{W}^{g e n} Q_{A}
$$

The r.h.s. of (6.4) is not only non-vanishing, but even worse it contains terms which are non-linear in the propagating fields (terms proportional to $\bar{c} \varphi_{1}, \bar{c} \varphi_{2}$ ):

$$
\begin{aligned}
\hat{W}^{g e n} Q_{(A)}=\int & \left\{-\partial_{\xi_{(A)}} z\left(Y_{2}+e \bar{c}\left(\hat{\varphi}_{1}-\xi_{A} \frac{m}{e}\right)\right)\left(\bar{\varphi}_{1}+\frac{\sqrt{z_{m}}}{\sqrt{z_{1}} z_{e}} \frac{m}{e}\right)\right. \\
& +\partial_{\xi_{(A)}} z^{-1}\left(Y_{1}-e \bar{c} \hat{\varphi}_{2}\right) \bar{\varphi}_{2} \\
& \left.+z \frac{m}{e}\left(\left(\partial_{\xi_{(A)}} \hat{\xi}_{A}-x \partial_{\xi_{(A)}} \xi_{A}\right) Y_{2}+\left(\partial_{\xi_{(A)}} \xi_{A}\right) e \bar{c}\left(\bar{\varphi}_{1}+\frac{\sqrt{z_{m}}}{\sqrt{z_{1}} z_{e}} \frac{m}{e}\right)\right)\right\}
\end{aligned}
$$

These non-linear terms are potentially harmful because they are not well-defined in higher orders. In order to overcome this difficulty we will absorb the harmful terms - in direct analogy to the treatment of [5] - into functional operators $\chi V^{g e n}$ and $\chi_{A} V_{A}^{g e n}$ which cancel these terms when acting on $\Gamma_{c l}^{g e n}$. A natural choice for $V^{g e n}$ and $V_{A}^{g e n}$ is given bys:

$$
V_{(A)}^{g e n}=\partial_{\xi_{(A)}} \int\left\{z\left(\hat{\varphi}_{1}-\xi_{A} \frac{m}{e}\right) \frac{\delta}{\delta q_{2}}-z^{-1} \hat{\varphi}_{2} \frac{\delta}{\delta q_{1}}\right\}
$$

An easy calculation now proves that the $\chi$ - and $\chi_{A}$-enlarged Ward operator

$$
W^{g e n}=\hat{W}^{g e n}+\chi V^{g e n}+\chi_{A} V_{A}^{g e n},
$$

\footnotetext{
${ }^{3}$ The expressions for $V^{g e n}$ and $V_{A}^{g e n}$ are most easily found by extending the operators in $\hat{W}^{g e n}$ as far as possible to invariant operators of the $\chi$ - and $\chi_{A}$-enlarged BRS transformations.
} 
when acting on $\Gamma_{c l}^{g e n}$, only leads to terms linear in the propagating fields:

$$
W^{g e n} \Gamma_{c l}^{g e n}=\chi \Delta_{b r}+\chi_{A} \Delta_{b r_{A}}
$$

with

$$
\Delta_{b r_{(A)}}=\partial_{\xi_{(A)}} \int\left\{z^{-1} Y_{1} \varphi_{2}-z Y_{2}\left(\varphi_{1}-\hat{\xi}_{A} \frac{m}{e}\right)\right\}
$$

The terms on the r.h.s. of (6.8) are harmless because they cannot be inserted non-trivially into higher orders' loop diagrams. This concludes the classical treatment.

Next we want to show that the WI (6.8) is valid in all orders of perturbation theory:

$$
W^{g e n} \Gamma=\chi \Delta_{b r}+\chi_{A} \Delta_{b r_{A}}
$$

$\Gamma$ now denotes the generating functional of 1-PI Green functions.

Because this proof almost completely parallels the proof given in [5] for one BRS transforming gauge parameter, we will concentrate on the essential steps only and skip some calculational details in between.

In order to work scheme-independently as far as possible when proving (6.10) we will only rely on the action principle whose validity has been shown in every renormalization scheme in use. This action principle implies:

$$
W^{g e n} \Gamma=\tilde{\Delta} \cdot \Gamma
$$

$\tilde{\Delta}$ is a local (i.e. field polynomial) integrated insertion carrying the quantum numbers: $\operatorname{dim} \tilde{\Delta} \leq 4, C(\tilde{\Delta}):-, \phi \pi(\tilde{\Delta})=0$.

The second ingredient, needed for the proof, is the transformation behaviour of $W^{\text {gen }}$ (6.7) under BRS transformations,

$$
\begin{aligned}
0= & W^{g e n} \mathcal{S}(\Gamma)=s_{\Gamma}\left(W^{g e n} \Gamma\right) \\
& -\left[\left(\chi \partial_{\xi}+\chi_{A} \partial_{\xi_{A}}\right) \int\left\{-z^{-1} \varphi_{2} \frac{\delta}{\delta \varphi_{1}}+z\left(\varphi_{1}-\hat{\xi}_{A} \frac{m}{e}\right) \frac{\delta}{\delta \varphi_{2}}-z Y_{2} \frac{\delta}{\delta Y_{1}}+z^{-1} Y_{1} \frac{\delta}{\delta Y_{2}}\right\}\right] \Gamma,
\end{aligned}
$$

where $s_{\Gamma}$ is given by (3.5).

Furthermore, another straightforward calculation yields:

$$
\begin{gathered}
s_{\Gamma}\left(\chi \Delta_{b r}+\chi_{A} \Delta_{b r_{A}}\right)= \\
+\left[\left(\chi \partial_{\xi}+\chi_{A} \partial_{\xi_{A}}\right) \int\left\{-z^{-1} \varphi_{2} \frac{\delta}{\delta \varphi_{1}}+z\left(\varphi_{1}-\hat{\xi}_{A} \frac{m}{e}\right) \frac{\delta}{\delta \varphi_{2}}-z Y_{2} \frac{\delta}{\delta Y_{1}}+z^{-1} Y_{1} \frac{\delta}{\delta Y_{2}}\right\}\right] \Gamma
\end{gathered}
$$

Hence combining (6.12) and (6.13) we find that the breaking of the WI (6.11) has to be $s_{\Gamma^{-} \text {-invariant: }}$

$$
s_{\Gamma}\left(\tilde{\Delta} \cdot \Gamma-\chi \Delta_{b r}-\chi_{A} \Delta_{b r_{A}}\right)=0
$$


From here on the proof of the $\chi$ - and $\chi_{A}$-enlarged Ward identity proceeds by induction in the loop expansion. In the tree approximation this WI has already been established (see above), and according to the action principle we have at 1-loop order:

$$
\left(W^{g e n} \Gamma\right)^{(\leq 1)}=\tilde{\Delta}^{(1)}
$$

Using the validity of the WI for $\chi=0=\chi_{A}\left(\right.$ 6.1] [9], $\tilde{\Delta}^{(\leq 1)}$ must have the form:

$$
\tilde{\Delta}^{(1)}=\chi\left(\Delta_{b r}^{(\leq 1)}+\Delta_{-}^{(1)}\right)+\chi_{A}\left(\Delta_{b r_{A}}^{(\leq 1)}+\Delta_{A-}^{(1)}\right)
$$

$\Delta_{-}^{(1)}, \Delta_{A-}^{(1)}$ are local insertions which carry $\phi \pi$-charge -1 , and due to the quantum numbers of the fields in question no term proportional to $\chi_{\chi_{A}}$ can appear in (6.16).

The application of $s_{\Gamma}$ to (6.15) then leads to

$$
s_{\Gamma}\left(\chi \Delta_{-}^{(1)}+\chi_{A} \Delta_{A-}^{(1)}\right)=s_{\Gamma_{c l}}\left(\chi \Delta_{-}^{(1)}+\chi_{A} \Delta_{A-}^{(1)}\right)+\mathcal{O}\left(\hbar^{2}\right)=0
$$

and hence we rest with a purely classical cohomology problem. This classical problem is solved as usual: First one has to find two bases of field polynomials constituting $\Delta_{-}^{(1)}$ and $\Delta_{A-}^{(1)}$, respectively $\left(v=\frac{m}{e}\right)$ :

$$
\begin{aligned}
\Delta_{(A)-}^{(1)}= & \int\left\{w_{(A) 1} Y_{1} \varphi_{2}+w_{(A) 2} Y_{1} \hat{\varphi}_{2}+w_{(A) 3} Y_{2}+w_{(A) 4} Y_{2} \varphi_{1}+w_{(A) 5} Y_{2} \hat{\varphi}_{1}\right. \\
& +w_{(A) 6} \bar{c}+w_{(A) 7}\left(-x Y_{2}+e \bar{c}\left(\bar{\varphi}_{1}+v\right)\right)+w_{(A) 8} \bar{c} \hat{\varphi}_{1}+w_{(A) 9} \bar{c} A^{2} \\
& +w_{(A) 10} \bar{c} \varphi_{1}^{2}+w_{(A) 11} \hat{\varphi}_{1}\left(-x Y_{2}+e \bar{c}\left(\bar{\varphi}_{1}+v\right)\right)+w_{(A) 12} \bar{c} \hat{\varphi}_{1}^{2}+w_{(A) 13} \bar{c} \varphi_{2}^{2} \\
& \left.+w_{(A) 14} \hat{\varphi}_{2}\left(-x Y_{1}-e \bar{c} \bar{\varphi}_{2}\right)+w_{(A) 15} \bar{c} \hat{\varphi}_{2}^{2}\right\}
\end{aligned}
$$

All the coefficients $w_{1}, \ldots, w_{15}, w_{A 1}, \ldots, w_{A 15}$ in (6.18) are of order $\hbar$.

In the next step the consistency condition (6.17) is used in order to determine the coefficients $w_{i}, w_{A i}$ as far as possible. Picking out in (6.17) terms proportional to $\chi$ we have

$$
\begin{aligned}
0= & \int\left(w_{1}\left(\varphi_{2} \frac{\delta \hat{\Gamma}_{c l}}{\delta \varphi_{1}}-Y_{1} \frac{\delta \hat{\Gamma}_{c l}}{\delta Y_{2}}\right)+w_{4}\left(\varphi_{1} \frac{\delta \hat{\Gamma}_{c l}}{\delta \varphi_{2}}-Y_{2} \frac{\delta \hat{\Gamma}_{c l}}{\delta Y_{1}}\right)+w_{3} \frac{\delta \hat{\Gamma}_{c l}}{\delta \varphi_{2}}\right. \\
& +w_{14}\left(\hat{\varphi}_{2} \frac{\delta \hat{\Gamma}_{c l}}{\delta \hat{\varphi}_{1}}+q_{2} \frac{\delta \hat{\Gamma}_{c l}}{\delta q_{1}}\right)+w_{11}\left(\hat{\varphi}_{1} \frac{\delta \hat{\Gamma}_{c l}}{\delta \hat{\varphi}_{2}}+q_{1} \frac{\delta \hat{\Gamma}_{c l}}{\delta q_{2}}\right)+w_{7} \frac{\delta \hat{\Gamma}_{c l}}{\delta \hat{\varphi}_{2}} \\
& +w_{2}\left(\hat{\varphi}_{2} \frac{\delta \hat{\Gamma}_{c l}}{\delta \varphi_{1}}-Y_{1} q_{2}\right)+w_{5}\left(\hat{\varphi}_{1} \frac{\delta \hat{\Gamma}_{c l}}{\delta \varphi_{2}}-Y_{2} q_{1}\right) \\
& +w_{6} B+w_{8}\left(B \hat{\varphi}_{1}-\bar{c} q_{1}\right)+w_{12}\left(B \hat{\varphi}_{1}^{2}-\bar{c} s \hat{\varphi}_{1}^{2}\right)+w_{15}\left(B \hat{\varphi}_{2}^{2}-\bar{c} s \hat{\varphi}_{2}^{2}\right) \\
& \left.+w_{9}\left(B A^{2}-\bar{c} s A^{2}\right)+w_{10}\left(B \varphi_{1}^{2}-\bar{c} s \varphi_{1}^{2}\right)+w_{13}\left(B \varphi_{2}^{2}-\bar{c} s \varphi_{2}^{2}\right)\right)
\end{aligned}
$$

and a quite analogous expression for the terms proportional to $\chi_{A}$ obtained from (6.19) by replacing all $w_{i}$ by the corresponding $w_{A i}$.

At this point we observe that the classical WI for $\chi=0=\chi_{A}$,

$$
\hat{W} \hat{\Gamma}_{c l}=0
$$


implies that not all of the polynomials in (6.19) can be independent. To proceed further,

we hence have to eliminate one of these polynomials via (6.20), for instance $\varphi_{2} \frac{\delta \hat{\Gamma}_{c l}}{\delta \varphi_{1}}-Y_{1} \frac{\delta \hat{\Gamma}_{c l}}{\delta Y_{2}}$. The remaining 14 polynomials are independent and therefore their coefficients have to vanish due to (6.19). In summary, algebraic considerations alone tell us that there possibly could exist a $\chi$ - and $\chi_{A}$-anomaly of the global WI (which nevertheless has to be $s_{\Gamma_{c l}}{ }^{-}$ invariant because of $(6.17))$ :

$$
\begin{aligned}
\left(W^{g e n} \Gamma\right)^{(\leq 1)}= & \chi \Delta_{b r}^{(\leq 1)}+\chi_{A} \Delta_{b r_{A}}^{(\leq 1)} \\
& +\left(\chi w_{1}+\chi_{A} w_{A 1}\right) \int\left(\left(Y_{1}-e \bar{c} \hat{\varphi}_{2}\right) \bar{\varphi}_{2}-\left(Y_{2}+e \bar{c}\left(\hat{\varphi}_{1}-\xi_{A} \frac{m}{e}\right)\right)\left(\bar{\varphi}_{1}+\frac{m}{e}\right)\right)
\end{aligned}
$$

Testing, however, (6.21) with respect to $Y_{1} \varphi_{2}$ and $Y_{2} \varphi_{1}$ and making use of the fact that the 3 -point functions disappear at an asymptotic momentum $p_{\infty}^{2}$,

$$
\begin{aligned}
& \Gamma_{Y_{1} \varphi_{1}}^{(1)}\left(p_{\infty}^{2}\right)+\Gamma_{Y_{2} \varphi_{2}}^{(1)}\left(p_{\infty}^{2}\right)=-\chi w_{1}-\chi_{A} w_{A 1} \\
& \Gamma_{Y_{1} \varphi_{1}}^{(1)}\left(p_{\infty}^{2}\right)+\Gamma_{Y_{2} \varphi_{2}}^{(1)}\left(p_{\infty}^{2}\right)=\chi w_{1}+\chi_{A} w_{A 1}
\end{aligned}
$$

we finally find:

$$
w_{1}=0 \quad \text { and } \quad w_{A 1}=0
$$

This concludes the proof of the $\chi$ - and $\chi_{A^{-}}$-enlarged WI at 1-loop order.

It is clear that this result can immediately be generalized to all orders of the perturbative expansion by repeating the reasoning just given when proving the induction step: order $n$ in $\hbar \longrightarrow$ order $n+1$ in $\hbar$. Hence we have shown (6.10) to all orders.

\section{The local Ward identity}

We conclude the first part of the present paper, which extends the results of [5] to the case when all gauge parameters of the model undergo BRS transformations by looking at the local Ward identity. This local WI governs the invariance of Green functions under (deformed) local gauge transformations and also yields information about the $\xi$ - and $\xi_{A^{-}}$ dependence of these Green functions. In analogy to the treatment of the global WI we again start with the local WI as it was proven in 9 for $\chi=0=\chi_{A}$ to all orders of perturbation theory,

$$
\left.\left((e+\delta e) w^{g e n}(x)-\partial_{\mu} \frac{\delta}{\delta A_{\mu}}\right) \Gamma\right|_{\chi=0=\chi_{A}}=\square B
$$


and then generalize to $\chi \neq 0$ and $\chi_{A} \neq 0$. In $(7.1) w^{g e n}(x)$ denotes the $\left(\chi^{-}\right.$and $\chi_{A^{-}}$ dependent) local Ward operator which is obtained from the global one (6.7) by taking away the integration,

$$
W^{g e n}=\int d^{4} x w^{g e n}(x),
$$

and $\delta e$ - to be fixed by the normalization condition for the coupling - is of order $\hbar$.

In the course of proving the $\chi$ - and $\chi_{A}$-dependent local WI it will turn out that the overall normalization factor of matter transformations $e+\delta e$ has to be independent of both gauge parameters $\xi$ and $\xi_{A}$ to all orders in the loop expansion. This fact will be the most important result of the actual investigation. It exactly reflects the restrictions

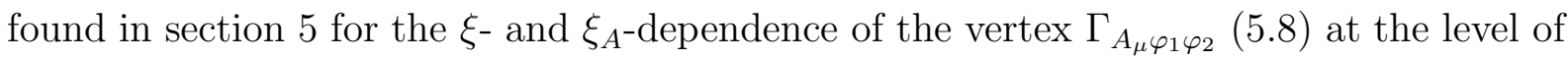
the local WI.

In the classical approximation a straightforward calculation shows that the following local WI holds true:

$$
\left(e w^{g e n}(x)-\partial_{\mu} \frac{\delta}{\delta A_{\mu}}\right) \Gamma_{c l}^{g e n}=\square B+e \chi D_{b r}(x)+e \chi_{A} D_{b r_{A}}(x)
$$

$D_{b r}(x)$ and $D_{b r_{A}}(x)$ are the (classical) non-integrated breaking terms $\Delta_{b r}$ and $\Delta_{b r_{A}}$ (6.9), respectively:

$$
\Delta_{b r_{(A)}}=\int d^{4} x D_{b r_{(A)}}(x)
$$

In order to proceed to higher orders we will make use of the same two, general, ingredients which allowed us to prove the global WI, namely the action principle and the transformation behaviour of the local Ward operator $w^{\text {gen }}(x)$ (7.2) under BRS transformations. Taking into account the validity of the global WI (6.10) and the local WI for $\chi=0=\chi_{A}$ the action principle implies that at 1-loop order we have:

$$
\begin{gathered}
\left.\left(\left(e+\delta e^{(1)}\right) w^{g e n}(x)-\partial_{\mu} \frac{\delta}{\delta A_{\mu}}\right) \Gamma\right|^{(\leq 1)}=\square B+\left(e+\delta e^{(1)}\right) \chi D_{b r}^{(\leq 1)}(x) \\
+\left(e+\delta e^{(1)}\right) \chi_{A} D_{b r_{A}}^{(\leq 1)}(x)+\chi \partial^{\mu} j_{\mu}^{(1)}+\chi_{A} \partial^{\mu} j_{A \mu}^{(1)}+\chi \chi_{A} \partial^{\mu} j_{\chi \chi_{A} \mu}^{(1)}
\end{gathered}
$$

The currents $j_{\mu}^{(1)}(x), j_{A \mu}^{(1)}(x)$ and $j_{\chi \chi_{A} \mu}^{(1)}(x)$ have dimension less than or equal to three and are odd under charge conjugation. Furthermore, $j_{\mu}^{(1)}(x)$ and $j_{A \mu}^{(1)}(x)$ carry $\phi \pi$-charge -1 , whereas $j_{\chi \chi_{A} \mu}^{(1)}(x)$ has $\phi \pi$-charge -2 . Looking at the quantum numbers of the fields in question we find that there is no possible term contributing to $j_{\chi \chi}^{(1)}{ }_{\mu}(x)$,

$$
j_{\chi \chi_{A} \mu}^{(1)}(x) \equiv 0
$$

and only one contribution to $j_{\mu}^{(1)}(x)$ and $j_{A \mu}^{(1)}(x)$, respectively:

$$
j_{\mu}^{(1)}(x)=u \partial_{\mu} \bar{c} \quad \text { and } \quad j_{A \mu}^{(1)}(x)=u_{A} \partial_{\mu} \bar{c}
$$


Next we have to exploit the transformation behaviour of $w^{g e n}(x)(7.2)$ under BRS transformations; in direct analogy to the corresponding considerations (6.12), (6.13) for the global Ward operator we deduce

$$
0=w^{g e n}(x) \mathcal{S}(\Gamma)=s_{\Gamma}\left(w^{g e n}(x) \Gamma-\chi D_{b r}(x)-\chi_{A} D_{b r_{A}}(x)\right)
$$

and also:

$$
0=\partial_{\mu} \frac{\delta}{\delta A_{\mu}} \mathcal{S}(\Gamma)=s_{\Gamma}\left(\partial_{\mu} \frac{\delta}{\delta A_{\mu}} \Gamma\right)
$$

Therefore, acting with the $\chi$ - and $\chi_{A}$-dependent $s_{\Gamma}$ on (7.5) and making use of the consistency condition (7.8) and (7.9) one obtains after a short calculation the following algebraic constraint:

$$
\begin{gathered}
\chi\left[\partial_{\xi}\left(e+\delta e^{(1)}\right)\right]\left(w^{g e n}(x) \Gamma-\chi_{A} D_{b r_{A}}(x)\right)+\chi_{A}\left[\partial_{\xi_{A}}\left(e+\delta e^{(1)}\right)\right]\left(w^{g e n}(x) \Gamma-\chi D_{b r}(x)\right) \\
=\chi \chi_{A}\left(\partial_{\xi} u_{A}-\partial_{\xi_{A}} u\right) \square \bar{c}-\chi u \square B-\chi_{A} u_{A} \square B
\end{gathered}
$$

Singling out in (7.10) terms proportional to $\chi$ or $\chi_{A}$, respectively, we find at 1-loop order:

$$
\begin{aligned}
\chi & :\left.\quad \partial_{\xi} \delta e^{(1)}\left(w^{g e n}(x) \Gamma\right)\right|_{\chi=0=\chi_{A}} ^{(0)}+u \square B=0 \\
\chi_{A} & :\left.\quad \partial_{\xi_{A}} \delta e^{(1)}\left(w^{g e n}(x) \Gamma\right)\right|_{\chi=0=\chi_{A}} ^{(0)}+u_{A} \square B=0
\end{aligned}
$$

Because the two insertions $\left.w^{g e n}(x) \Gamma\right|_{\chi=0=\chi_{A}} ^{(0)}$ and $\square B$ are linearly independent (as can be seen by directly calculating $\left.w^{g e n}(x) \Gamma\right|_{\chi=0=\chi_{A}} ^{(0)}$, see also [5]) the coefficients in front of these two insertions have to vanish separately, i.e.:

$$
u=0=u_{A} \quad \text { and } \quad \partial_{\xi} \delta e^{(1)}=0=\partial_{\xi_{A}} \delta e^{(1)}
$$

Inserting $u=0=u_{A}$ into (7.5) completes the proof of the local WI at 1-loop order.

It is obvious that the argument just given can be extended to all orders of perturbation theory by induction. Hence we finally end up with the following $\chi_{\text {- }}$ and $\chi_{A}$-dependent local WI:

$$
\left((e+\delta e) w^{g e n}(x)-\partial_{\mu} \frac{\delta}{\delta A_{\mu}}\right) \Gamma=\square B+(e+\delta e) \chi D_{b r}(x)+(e+\delta e) \chi_{A} D_{b r_{A}}(x)
$$

In addition we have shown that the overall normalization factor of the matter transformations has to be $\xi$ - and $\xi_{A}$-independent in all orders of the perturbative expansion:

$$
\partial_{\xi}(e+\delta e)=0=\partial_{\xi_{A}}(e+\delta e)
$$

This result is highly non-trivial and can be obtained in this generality only with the formalism of BRS transforming gauge parameters.

As already mentioned above the constraint (7.15) found at the level of the local WI is the 
direct analogue of the restriction (5.8) which we derived for the $\xi$ - and $\xi_{A}$-dependence of the vertex $\Gamma_{A_{\mu} \varphi_{1} \varphi_{2}}$. In section 5 we also discussed that this restriction carefully has to be taken into account when a normalization condition for the coupling is formulated, leading in section 5 to the introduction of two additional parameters into the theory, namely the two reference points $\xi_{0}$ and $\xi_{A_{0}}$, see (5.9). The normalization condition (5.9), however, poses quite troublesome difficulties when explicit calculations are to be performed.

But, having proven (7.15) we have at hand a new possibility for fixing the couplingf: Following the line of argument, the normalization condition for the coupling has to respect the $\xi$ - and $\xi_{A}$-independence of the factor $e+\delta e$. This is trivially fulfilled if we demand $\delta e=0$, i.e. if we require the local WI to be exact to all orders of perturbation theory:

$$
\left.\left(e w^{g e n}(x)-\partial_{\mu} \frac{\delta}{\delta A_{\mu}}\right) \Gamma\right|_{\chi=0=\chi_{A}}=\square B
$$

The normalization condition (7.16) (replacing (5.9)) is much easier manageable in concrete calculations.

In summary, we have shown that the on-shell normalization conditions taken together with the requirement "local WI exact to all orders" are in agreement with the $\chi$ - and $\chi_{A^{-}}$ enlarged ST identity and hence guarantuee a correct treatment of full gauge parameter dependence in explicit calculations.

\section{BRS-symmetric insertions}

As an application of the general formalism developed so far we want to study parametric differential equations of the type

$$
\lambda \partial_{\lambda} \Gamma=\Delta_{\lambda} \cdot \Gamma
$$

in the next section, where $\lambda$ denotes a (set of) parameter(s) of the theory. Due to the action principle $\Delta_{\lambda}$ is an insertion of dimension less than or equal to four, even under charge conjugation and BRS invariant. This last property holds because of

$$
0=\lambda \partial_{\lambda} \mathcal{S}(\Gamma)=s_{\Gamma}\left(\lambda \partial_{\lambda} \Gamma\right)=s_{\Gamma}\left(\Delta_{\lambda} \cdot \Gamma\right)=s_{\Gamma_{c l}} \Delta_{\lambda}+\mathcal{O}(\hbar)
$$

for $\lambda$ being independent of $\xi$ and $\xi_{A}$. Therefore, as a preparatory step we first have to classify all BRS-symmetric insertions, which carry the same quantum numbers as $\Gamma$. Because in the present paper we are mainly interested in questions concerning gauge parameter

\footnotetext{
${ }^{4}$ See also [5] for a more detailed discussion.
} 
dependence we will pay special attention to the appearing of $\xi$ - and $\xi_{A}$-dependence.

In order to solve the cohomological problem mentioned above we once more return to the classical level and write down all independent field polynomials fulfilling

$$
s_{\Gamma_{c l}} \Delta_{\lambda}=0
$$

Then we have to translate these polynomials to BRS-invariant operators, only this last representation being valid to all orders of perturbation theory. Because the solution of this problem for $\chi=0=\chi_{A}$ was already given in [9] we will make use of the following trick to handle the modifications for $\chi \neq 0, \chi_{A} \neq 0$ :

First we decompose $\Delta_{\lambda}$ into three parts by explicitly separating $\chi$ - and $\chi_{A}$-dependence:

$$
\Delta_{\lambda}=\Delta_{\lambda}^{0}+\chi \Delta_{\lambda}^{-}+\chi_{A} \Delta_{A, \lambda}^{-}
$$

(Please note that due to the quantum numbers of $\Delta_{\lambda}$ no term proportional to $\chi_{A}$ can appear.)

Splitting $\Gamma_{c l}=\hat{\Gamma}_{c l}+\chi Q+\chi_{A} Q_{A}$ and $s_{\Gamma_{c l}}$ in the same way,

$$
\begin{aligned}
s_{\Gamma_{c l}} & =s_{\hat{\Gamma}_{c l}=0=\chi_{A}}+\chi\left(\partial_{\xi}+\mathcal{O}\right)+\chi_{A}\left(\partial_{\xi_{A}}+\mathcal{O}_{A}\right), \\
\text { with } \mathcal{O} & =\int\left\{\frac{\delta Q}{\delta \underline{\varphi}} \frac{\delta}{\delta \underline{Y}}-\frac{\delta Q}{\delta \underline{Y}} \frac{\delta}{\delta \underline{\varphi}}\right\}, \\
\mathcal{O}_{A} & =\int\left\{\frac{\delta Q_{A}}{\delta \underline{\varphi}} \frac{\delta}{\delta \underline{Y}}-\frac{\delta Q_{A}}{\delta \underline{Y}} \frac{\delta}{\delta \underline{\varphi}}\right\},
\end{aligned}
$$

we find that $(8.3)$ is equivalent to the following four equations:

$$
\begin{aligned}
s_{\hat{\Gamma}_{c l}^{\chi=0=\chi_{A}} \Delta_{\lambda}^{0}}^{\chi=} & =0 \\
s_{\hat{\Gamma}_{c l}^{\chi=0=\chi_{A}} \Delta_{\lambda}^{-}}^{-} & =\left(\partial_{\xi}+\mathcal{O}\right) \Delta_{\lambda}^{0} \\
s_{\hat{\Gamma}_{c l}=0=\chi_{A}} \Delta_{A, \lambda}^{-} & =\left(\partial_{\xi_{A}}+\mathcal{O}_{A}\right) \Delta_{\lambda}^{0} \\
\left(\partial_{\xi}+\mathcal{O}\right) \Delta_{A, \lambda}^{-} & =\left(\partial_{\xi_{A}}+\mathcal{O}_{A}\right) \Delta_{\lambda}^{-}
\end{aligned}
$$

Now it is easy to see that it is always possible to find a $\hat{\Delta}_{\lambda}^{-}$such that:

$$
\Delta_{\lambda}^{-}=\left(\partial_{\xi}+\mathcal{O}\right) \hat{\Delta}_{\lambda}^{-} \quad \text { and } \quad \Delta_{A, \lambda}^{-}=\left(\partial_{\xi_{A}}+\mathcal{O}_{A}\right) \hat{\Delta}_{\lambda}^{-}
$$

We remark that due to 8 8.10) eq. (8.9) is fulfilled automatically.

With these preparations we have

$$
\begin{aligned}
\left(\partial_{\xi}+\mathcal{O}\right)\left(\Delta_{\lambda}^{0}-s_{\hat{\Gamma}_{c l}}^{\chi=0=\chi_{A}} \hat{\Delta}_{\lambda}^{-}\right) & =s_{\hat{\Gamma}_{c l}=0=\chi_{A}} \Delta_{\lambda}^{-}-\left(\partial_{\xi}+\mathcal{O}\right) s_{\hat{\Gamma}_{c l}=0=\chi_{A}}^{\chi \Delta_{\lambda}^{-}} \\
& =s_{\hat{\Gamma}_{c l}=0=\chi_{A}} \Delta_{\lambda}^{-}-s_{\hat{\Gamma}_{c l}=0=\chi_{A}}^{\chi=}\left(\partial_{\xi}+\mathcal{O}\right) \hat{\Delta}_{\lambda}^{-}=0
\end{aligned}
$$


and in the same way

$$
\left(\partial_{\xi_{A}}+\mathcal{O}_{A}\right)\left(\Delta_{\lambda}^{0}-s_{\hat{\Gamma}_{c l}}^{\chi=0=\chi_{A}} \hat{\Delta}_{\lambda}^{-}\right)=0
$$

But that means

$$
\Delta_{\lambda}^{0}=s_{\hat{\Gamma}_{c l}}^{\chi=0=\chi_{A}} \hat{\Delta}_{\lambda}^{-}+\hat{\Delta}_{\lambda}^{0}
$$

with

$$
\left(\partial_{\xi}+\mathcal{O}\right) \hat{\Delta}_{\lambda}^{0}=0=\left(\partial_{\xi_{A}}+\mathcal{O}_{A}\right) \hat{\Delta}_{\lambda}^{0}
$$

and

$$
s_{\hat{\Gamma}_{c l}}^{\chi=0=\chi_{A}} \hat{\Delta}_{\lambda}^{0}=0
$$

Hence:

$$
\begin{aligned}
\Delta_{\lambda} & =\Delta_{\lambda}^{0}+\chi \Delta_{\lambda}^{-}+\chi_{A} \Delta_{A, \lambda}^{-} \\
& =s_{\hat{\Gamma}_{c l}=\chi_{A}}^{\chi} \hat{\Delta}_{\lambda}^{-}+\hat{\Delta}_{\lambda}^{0}+\chi\left(\partial_{\xi}+\mathcal{O}\right) \hat{\Delta}_{\lambda}^{-}+\chi_{A}\left(\partial_{\xi_{A}}+\mathcal{O}_{A}\right) \hat{\Delta}_{\lambda}^{-} \\
& =\hat{\Delta}_{\lambda}^{0}+s_{\Gamma_{c l}} \hat{\Delta}_{\lambda}^{-}
\end{aligned}
$$

As already mentioned, the solution of (8.15) was presented in [9] and we just give the list of terms contributing to $\hat{\Delta}_{\lambda}^{0}$ in appendix B. The crucial point in this context is, however: A short calculation starting from (8.14) shows that all terms in $\hat{\Delta}_{\lambda}^{0}$ which are no BRS variations, namely冋,

$$
\int \frac{\delta \hat{\Gamma}_{c l}}{\delta \hat{\varphi}_{0}}, \int\left\{A \frac{\delta}{\delta A}+c \frac{\delta}{\delta c}\right\} \hat{\Gamma}_{c l}, m_{H} \partial_{m_{H}} \hat{\Gamma}_{c l}, e \partial_{e} \hat{\Gamma}_{c l}
$$

have to appear with coefficients which are independent of $\xi$ and $\xi_{A}$.

For $\hat{\Delta}_{\lambda}^{-}$we choose the most general ansatz compatible with the quantum numbers of $\hat{\Delta}_{\lambda}^{-}$ ( $\phi \pi$-charge: $-1, C:+$, dim: $\leq 4$ ), see also (4.8); in view of the generalization to higher orders, this ansatz can be brought into the form:

$$
\begin{aligned}
\hat{\Delta}_{\lambda}^{-}: & \int Y_{1}, \frac{\delta \hat{\Gamma}_{c l}}{\delta q_{1}}, \bar{c} \hat{\varphi}_{2}, \\
& Y_{1} \varphi_{1}, Y_{1} \hat{\varphi}_{1}, Y_{2} \varphi_{2}, Y_{2} \hat{\varphi}_{2}, \bar{c} \frac{\delta \hat{\Gamma}_{c l}}{\delta B}, \hat{\varphi}_{i} \frac{\delta \hat{\Gamma}_{c l}}{\delta q_{i}}, \bar{c} \hat{\varphi}_{1} \hat{\varphi}_{2}, \bar{c} B
\end{aligned}
$$

Looking to (B.1) $-(\overline{B .4})$ and (8.16), we find that all terms in $\hat{\Delta}_{\lambda}^{0}$ which are BRS variations have to be modified when BRS transforming gauge parameters are included. Therefore

\footnotetext{
${ }^{5}$ The definition of the additional external field $\hat{\varphi}_{0}$ is also given in appendix B.
} 
we finally end up with the following basis of BRS invariant insertions which we directly give in the form of BRS invariant operators $(i=1,2)$ :

$$
\begin{aligned}
& f_{s, 0} \int \frac{\delta \Gamma}{\delta \varphi_{1}}+\left(\chi \partial_{\xi}+\chi_{A} \partial_{\xi_{A}}\right) f_{s, 0} \int Y_{1}=s_{\Gamma}\left(f_{s, 0} \int Y_{1}\right) \\
& \hat{f}_{s, 0} \int \frac{\delta \Gamma}{\delta \hat{\varphi}_{1}}+\left[\left(\chi \partial_{\xi}+\chi_{A} \partial_{\xi_{A}}\right) \hat{f}_{s, 0}\right] \int \frac{\delta \Gamma}{\delta q_{1}}=s_{\Gamma}\left(\hat{f}_{s, 0} \int \frac{\delta \Gamma}{\delta q_{1}}\right) \\
& \tilde{f}_{3} \int\left\{B \hat{\varphi}_{2}-\bar{c} q_{2}\right\}+\left(\chi \partial_{\xi}+\chi_{A} \partial_{\xi_{A}}\right) \tilde{f}_{3} \int \bar{c} \hat{\varphi}_{2}=s_{\Gamma}\left(\tilde{f}_{3} \int \bar{c} \hat{\varphi}_{2}\right) \\
& \mathcal{N}_{s, i}^{\left(\chi, \chi_{A}\right)} \Gamma \equiv f_{s, i} \int\left\{\varphi_{i} \frac{\delta}{\delta \varphi_{i}}-Y_{i} \frac{\delta}{\delta Y_{i}}\right\} \Gamma+\left(\chi \partial_{\xi}+\chi_{A} \partial_{\xi_{A}}\right) f_{s, i} \int Y_{i} \varphi_{i}=s_{\Gamma}\left(f_{s, i} \int Y_{i} \varphi_{i}\right) \\
& \hat{\mathcal{N}}_{s, i}^{\left(\chi, \chi_{A}\right)} \Gamma \equiv \hat{f}_{s, i} \int\left\{q_{i} \frac{\delta}{\delta q_{i}}+\hat{\varphi}_{i} \frac{\delta}{\delta \hat{\varphi}_{i}}\right\} \Gamma+\left[\left(\chi \partial_{\xi}+\chi_{A} \partial_{\xi_{A}}\right) \hat{f}_{s, i}\right] \int \hat{\varphi}_{i} \frac{\delta \Gamma}{\delta q_{i}}=s_{\Gamma}\left(\hat{f}_{s, i} \int \hat{\varphi}_{i} \frac{\delta \Gamma}{\delta q_{i}}\right) \\
& \overline{\mathcal{N}}_{s, i}^{\left(\chi, \chi_{A}\right)} \Gamma \equiv \bar{f}_{s, i} \int\left\{\hat{\varphi}_{i} \frac{\delta \Gamma}{\delta \varphi_{i}}-Y_{i} q_{i}\right\}+\left(\chi \partial_{\xi}+\chi_{A} \partial_{\xi_{A}}\right) \bar{f}_{s, i} \int Y_{i} \hat{\varphi}_{i}=s_{\Gamma}\left(\bar{f}_{s, i} \int Y_{i} \hat{\varphi}_{i}\right) \\
& \mathcal{N}_{B}^{\left(\chi, \chi_{A}\right)} \Gamma \equiv f_{B} \int\left\{B \frac{\delta}{\delta B}+\bar{c} \frac{\delta}{\delta \bar{c}}\right\} \Gamma+\left[\left(\chi \partial_{\xi}+\chi_{A} \partial_{\xi_{A}}\right) f_{B}\right] \int \bar{c} \frac{\delta \Gamma}{\delta B}=s_{\Gamma}\left(f_{B} \int \bar{c} \frac{\delta \Gamma}{\delta B}\right) \\
& \tilde{f}_{4} \int\left\{B \hat{\varphi}_{1} \hat{\varphi}_{2}-\bar{c} q_{1} \hat{\varphi}_{2}-\bar{c} \hat{\varphi}_{1} q_{2}\right\}+\left(\chi \partial_{\xi}+\chi_{A} \partial_{\xi_{A}}\right) \tilde{f}_{4} \int \bar{c} \hat{\varphi}_{1} \hat{\varphi}_{2}=s_{\Gamma}\left(\tilde{f}_{4} \int \bar{c} \hat{\varphi}_{1} \hat{\varphi}_{2}\right) \\
& f_{\xi} \partial_{\xi} \Gamma+\left[\left(\chi \partial_{\xi}+\chi \chi_{A} \partial_{\xi_{A}}\right) f_{\xi}\right] \partial_{\chi} \Gamma=s_{\Gamma}\left(f_{\xi} \partial_{\chi} \Gamma\right)
\end{aligned}
$$

In addition there are the BRS symmetric operators from (8.17) (with $\hat{\Gamma}_{c l}$ replaced by $\Gamma$ ).

\section{Parametric differential equations}

Having finished the preparatory considerations dealing with the BRS symmetric insertions we now can turn to the derivation of some partial differential equations, namely the Callan-Symanzik (CS) equation and the renormalization group (RG) equation. We will also comment about the dependence of the theory on the ghost mass which is governed by the differential operator $\xi_{A} \partial_{\xi_{A}}$ due to the normalization condition (2.17).

\subsection{CS equation}

The CS equation describes the response of the system to the scaling of all independent parameters carrying dimension of mass. In the model under investigation the CS operator is hence given by

$$
\underline{m} \partial_{\underline{m}} \equiv m \partial_{m}+m_{H} \partial_{m_{H}}+\kappa \partial_{\kappa}
$$


and we have the task to construct the r.h.s. of $\underline{m} \partial_{\underline{m}} \Gamma=$ ? which according to the action principle

$$
\underline{m} \partial_{\underline{m}} \Gamma=\Delta_{m} \cdot \Gamma
$$

has to be an insertion with dimension less than or equal to four, even under charge conjugation and also BRS invariant. (I.e. $\underline{m} \partial_{\underline{m}}$ is an operator of the type $\lambda \partial_{\lambda}$ discussed in the previous section.) In [9] it was shown that in order to construct a unique r.h.s. of the CS equation rigid invariance has to be used, too. Therefore we next calculate the commutator of the $\chi$ - and $\chi_{A}$-enlarged global Ward operator (6.7) and $\underline{m} \underline{\partial}_{\underline{m}}$ :

$$
\left[W^{g e n}, \underline{m} \partial_{\underline{m}}\right]=z \int\left\{\hat{\xi}_{A} \frac{m}{e} \frac{\delta}{\delta \varphi_{2}}+\xi_{A} \frac{m}{e} \frac{\delta}{\delta \hat{\varphi}_{2}}\right\}+\left(\chi \partial_{\xi}+\chi_{A} \partial_{\xi_{A}}\right) z \xi_{A} \frac{m}{e} \int \frac{\delta}{\delta q_{2}}
$$

In order to make the line of argument as transparent as possible and to explicitly work out what is needed in the following we introduce the $W^{g e n}$-symmetric extension $\underline{m} \tilde{\partial}_{\underline{m}}$ of $\underline{m} \partial_{\underline{m}}$ originating from $(\underline{9.3})$,

$$
\begin{gathered}
\underline{m} \tilde{\partial}_{\underline{m}} \equiv \underline{m} \partial_{\underline{m}}+\hat{\xi}_{A} \frac{m}{e} \int \frac{\delta}{\delta \varphi_{1}}+\xi_{A} \frac{m}{e} \int \frac{\delta}{\delta \hat{\varphi}_{1}}+\left(\chi \partial_{\xi}+\chi_{A} \partial_{\xi_{A}}\right) \xi_{A} \frac{m}{e} \int \frac{\delta}{\delta q_{1}} \\
{\left[W^{g e n}, \underline{m} \tilde{\partial}_{\underline{m}}\right]=0,}
\end{gathered}
$$

and consider the insertion $\underline{m} \tilde{\partial}_{\underline{m}} \Gamma=\tilde{\Delta}_{m} \cdot \Gamma$ instead of $\underline{m} \partial_{\underline{m}} \Gamma=\Delta_{m} \cdot \Gamma$. Due to (9.5) we have:

$$
W^{g e n}\left(\tilde{\Delta}_{m} \cdot \Gamma\right)=\underline{m} \underline{\partial}_{\underline{m}} W^{g e n} \Gamma=\underline{m} \underline{\tilde{\partial}}_{\underline{m}}\left(\chi \Delta_{b r}+\chi_{A} \Delta_{b r_{A}}\right)=z\left(\chi \partial_{\xi}+\chi_{A} \partial_{\xi_{A}}\right) \hat{\xi}_{A} \frac{m}{e} \int Y_{2}
$$

Please note that the application of $W^{g e n}$ to the term

$$
-\left(\chi \partial_{\xi}+\chi_{A} \partial_{\xi_{A}}\right) \hat{\xi}_{A} \frac{m}{e} \int Y_{1}
$$

this term being part of the first insertion in (8.19), exactly cancels the r.h.s. of (9.6). Therefore, all other BRS symmetric ${ }^{6}$ insertions building up $\tilde{\Delta}_{m} \cdot \Gamma$ have to be symmetrized with respect to $W^{g e n}$ : Only in this $W^{g e n}$-symmetrized form they can contribute to the r.h.s. of the CS equation. For some of the operators in (8.17), (8.19), (8.20) (namely the $\left(\chi\right.$ - and $\chi_{A}$-enlarged) leg counting operators) this symmetrization can be achieved easily:

$$
\begin{aligned}
\mathcal{N}_{s}^{\left(\chi, \chi_{A}\right)} \Gamma & \equiv f_{s} N_{s} \Gamma-f_{s} \hat{\xi}_{A} \frac{m}{e} \int \frac{\delta \Gamma}{\delta \varphi_{1}}+\left(\chi \partial_{\xi}+\chi_{A} \partial_{\xi_{A}}\right) f_{s} \int\left\{Y_{1}\left(\varphi_{1}-\hat{\xi}_{A} \frac{m}{e}\right)+Y_{2} \varphi_{2}\right\} \\
\hat{\mathcal{N}}_{s}^{\left(\chi, \chi_{A}\right)} & \equiv \hat{f}_{s} \hat{N}_{s}-\hat{f}_{s} \xi_{A} \frac{m}{e} \int \frac{\delta}{\delta \hat{\varphi}_{1}}+\left(\chi \partial_{\xi}+\chi_{A} \partial_{\xi_{A}}\right) \hat{f}_{s} \int\left\{\left(\hat{\varphi}_{1}-\xi_{A} \frac{m}{e}\right) \frac{\delta}{\delta q_{1}}+\hat{\varphi}_{2} \frac{\delta}{\delta q_{2}}\right\},
\end{aligned}
$$

\footnotetext{
${ }^{6}$ The operators extending $\underline{m} \partial_{\underline{m}}$ in (9.4) taken together with (9.7) (times -1 ) just constitute the first two BRS symmetric insertions in $(8.19)$; hence the remaining contributions to $\tilde{\Delta}_{m} \cdot \Gamma$ have to be BRS symmetric.
} 


$$
\begin{aligned}
N_{A} & \equiv \int\left\{A \frac{\delta}{\delta A}+c \frac{\delta}{\delta c}\right\}, \\
\mathcal{N}_{B}^{\left(\chi, \chi_{A}\right)} & \equiv f_{B} N_{B}+\left(\chi \partial_{\xi}+\chi_{A} \partial_{\xi_{A}}\right) f_{B} \int \bar{c} \frac{\delta}{\delta B}
\end{aligned}
$$

The mixed operators containing $\hat{\varphi}_{i} \frac{\delta \Gamma}{\delta \varphi_{i}}$ are symmetrized like the leg counting operators:

$$
\begin{aligned}
\overline{\mathcal{N}}_{s}^{\left(\chi, \chi_{A}\right)} \Gamma \equiv & \bar{f}_{s} \bar{N}_{s} \Gamma-\bar{f}_{s} \xi_{A} \frac{m}{e} \int \frac{\delta \Gamma}{\delta \varphi_{1}}+\bar{f}_{s} \int\left\{q_{1} Y_{1}+q_{2} Y_{2}\right\} \\
& +\left(\chi \partial_{\xi}+\chi_{A} \partial_{\xi_{A}}\right) \bar{f}_{s} \int\left\{Y_{1}\left(\hat{\varphi}_{1}-\xi_{A} \frac{m}{e}\right)+Y_{2} \hat{\varphi}_{2}\right\}
\end{aligned}
$$

In (9.8), (9.9) we have introduced the usual leg counting operators:

$$
\begin{aligned}
N_{s} & \equiv \int\left\{\varphi_{1} \frac{\delta}{\delta \varphi_{1}}+\varphi_{2} \frac{\delta}{\delta \varphi_{2}}-Y_{1} \frac{\delta}{\delta Y_{1}}-Y_{2} \frac{\delta}{\delta Y_{2}}\right\}, \\
\hat{N}_{s} & \equiv \int\left\{\hat{\varphi}_{1} \frac{\delta}{\delta \hat{\varphi}_{1}}+\hat{\varphi}_{2} \frac{\delta}{\delta \hat{\varphi}_{2}}+q_{1} \frac{\delta}{\delta q_{1}}+q_{2} \frac{\delta}{\delta q_{2}}\right\}, \\
\bar{N}_{s} & \equiv \int\left\{\hat{\varphi}_{1} \frac{\delta}{\delta \varphi_{1}}+\hat{\varphi}_{2} \frac{\delta}{\delta \varphi_{2}}\right\}, \\
N_{B} & \equiv \int\left\{B \frac{\delta}{\delta B}+\bar{c} \frac{\delta}{\delta \bar{c}}\right\}
\end{aligned}
$$

To find the $W^{g e n}$-symmetric extensions of the differential operators $m_{H} \partial_{m_{H}}, e \partial_{e}$ and the operator containing $\partial_{\xi}$ (see last line of (8.20)),

$$
m_{H} \partial_{m_{H}} \rightarrow m_{H} \tilde{\partial}_{m_{H}} \quad, \quad e \partial_{e} \rightarrow e \tilde{\partial}_{e} \quad, \quad f_{\xi} \partial_{\xi}+\left(\chi \partial_{\xi}+\chi_{A} \partial_{\xi_{A}}\right) f_{\xi} \partial_{\chi} \rightarrow \tilde{\partial}_{\xi}
$$

indeed requires some calculation. The final expressions being rather lengthy we present the explicit results of the symmetrization in appendix C.

Finally we observe that the insertion $\frac{\delta}{\delta \hat{\varphi}_{0}}$ already is $W^{g e n}$-symmetric and that the remaining two insertions in (8.19), (8.20) cannot be extended in a $W^{g e n}$-symmetric way.

Thus the final answer is: (9.8), (9.9), (C.5) and $\frac{\delta}{\delta \hat{\varphi}_{0}}$ provide a basis of BRS symmetric and rigidly invariant operators which are even under charge conjugation and have dimension less than or equal to four. Hence the insertion $\tilde{\Delta}_{m} \cdot \Gamma$ can be decomposed as follows:

$$
\begin{aligned}
\widetilde{\mathcal{C}} \Gamma \equiv & \left(\underline{m} \tilde{\partial}_{\underline{m}}+\beta_{e} e \tilde{\partial}_{e}+\beta_{m_{H}} m_{H} \tilde{\partial}_{m_{H}}+\tilde{\beta}_{\xi} \tilde{\partial}_{\xi}\right. \\
& \left.-\tilde{\gamma}_{s} \mathcal{N}_{s}^{\left(\chi, \chi_{A}\right)}-\tilde{\hat{\gamma}}_{s} \hat{\mathcal{N}}_{s}^{\left(\chi, \chi_{A}\right)}-\tilde{\bar{\gamma}}_{s} \overline{\mathcal{N}}_{s}^{\left(\chi, \chi_{A}\right)}-\gamma_{A} N_{A}-\tilde{\gamma}_{B} \mathcal{N}_{B}^{\left(\chi, \chi_{A}\right)}-\alpha_{i n v} \int \delta_{\hat{\varphi}_{0}}\right) \Gamma \\
= & -\left(\chi \partial_{\xi}+\chi_{A} \partial_{\xi_{A}}\right) \hat{\xi}_{A} \frac{m}{e} \int Y_{1}
\end{aligned}
$$

Equation (9.12) is the CS equation in the manifestly $W^{g e n}$-symmetric form. The important result in our context is that the $\beta$-functions $\beta_{e}$ and $\beta_{m_{H}}$ as well as the anomalous 
dimension $\gamma_{A}$ and $\alpha_{i n v}$ are independent of both the gauge parameters $\xi$ and $\xi_{A}$ to all orders of perturbation theory. The coefficient functions $\tilde{\beta}_{\xi}, \tilde{\gamma}_{s}, \tilde{\hat{\gamma}}_{s}, \tilde{\bar{\gamma}}_{s}, \tilde{\gamma}_{B}$ also are $\xi$ - and $\xi_{A}$-independent, but the usual (i.e. complete) $\beta$ - and $\gamma$-functions

$$
\beta_{\xi} \xi=\tilde{\beta}_{\xi} f_{\xi}, \gamma_{s}=\tilde{\gamma}_{s} f_{s}, \hat{\gamma}_{s}=\tilde{\hat{\gamma}}_{s} \hat{f}_{s}, \bar{\gamma}_{s}=\tilde{\bar{\gamma}}_{s} \bar{f}_{s}, \gamma_{B}=\tilde{\gamma}_{B} f_{B}
$$

a priori may depend on both the gauge parameters $\xi$ and $\xi_{A}$ through the factors $f_{(s, \xi)}\left(\xi, \xi_{A}\right)$ appearing in the leg counting operators $\mathcal{N}_{s}^{\left(\chi, \chi_{A}\right)}(9.8),(9.9)$ and in $\tilde{\partial}_{\xi}$ (C.5).

So far one can get with symmetry considerations alone. If additional information about the coefficient functions is requested one has to test (9.12) on the gauge condition (4.2), to make use of the local WI (7.14) and/or to carry out explicit calculations: Testing (9.12) on the gauge condition (4.2) we find

$$
\begin{aligned}
\gamma_{B} & =-\gamma_{A} \\
\beta_{\xi}=2 \gamma_{B} & =-2 \gamma_{A} \\
\beta_{e}+\gamma_{A}-\gamma_{s}-\hat{\gamma}_{s} & =\left(\beta_{e} e \partial_{e}+\beta_{m_{H}} m_{H} \partial_{m_{H}}-2 \gamma_{A} \xi \partial_{\xi}\right) \ln z
\end{aligned}
$$

and hence also $\gamma_{B}$ and $\beta_{\xi}$ are completely gauge parameter-independent to all orders. Furthermore, using the validity of the local WI (7.14) and the normalization condition for the coupling (7.16) yields (see [9] for details):

$$
\gamma_{A}=\beta_{e}
$$

We want to conclude this subsection by rewriting the CS equation in its much more convenient form which separates the hard and soft breaking on the left and right hand side of the CS equation:

$$
\begin{aligned}
& \left(\underline{m} \partial_{\underline{m}}+\beta_{e} e \partial_{e}+\beta_{m_{H}} m_{H} \partial_{m_{H}}-\gamma_{s} N_{s}-\hat{\gamma}_{s} \hat{N}_{s}-\bar{\gamma}_{s} \bar{N}_{s}-\beta_{e}\left(N_{A}-N_{B}+2 \xi \partial_{\xi}\right)\right. \\
& -\gamma_{1} \int\left\{\varphi_{1} \frac{\delta}{\delta \varphi_{1}}-Y_{1} \frac{\delta}{\delta Y_{1}}+\hat{\varphi}_{1} \frac{\delta}{\delta \hat{\varphi}_{1}}+q_{1} \frac{\delta}{\delta q_{1}}\right\} \\
& \left.-\left(\chi \partial_{\xi}+\chi_{A} \partial_{\xi_{A}}\right) \int\left\{-\gamma_{s} \hat{\varphi}_{1} \frac{\delta}{\delta q_{1}}+\hat{\gamma}_{s} \hat{\varphi}_{2} \frac{\delta}{\delta q_{2}}\right\}-2 \beta_{e} \chi \partial_{\chi}\right) \Gamma \\
= & -\frac{m}{e} \int\left\{\left(\hat{\xi}_{A}+\alpha_{1}\right) \frac{\delta}{\delta \varphi_{1}}+\left(\xi_{A}+\hat{\alpha}_{1}\right) \frac{\delta}{\delta \hat{\varphi}_{1}}-\alpha_{i n v} \frac{e}{m} \frac{\delta}{\delta \hat{\varphi}_{0}}+\left(\chi \partial_{\xi}+\chi_{A} \partial_{\xi_{A}}\right)\left(\xi_{A}+\hat{\alpha}_{1}\right) \frac{\delta}{\delta q_{1}}\right\} \Gamma \\
& +\bar{\gamma}_{s} \int\left\{q_{1} Y_{1}+q_{2} Y_{2}\right\} \\
& +\left(\chi \partial_{\xi}+\chi_{A} \partial_{\xi_{A}}\right) \int\left\{\left(\gamma_{s}+\gamma_{1}\right) Y_{1} \varphi_{1}+\gamma_{s} Y_{2} \varphi_{2}-\frac{m}{e}\left(\hat{\xi}_{A}+\alpha_{1}\right) Y_{1}+\bar{\gamma}_{s}\left(Y_{1} \hat{\varphi}_{1}+Y_{2} \hat{\varphi}_{2}\right)\right\}
\end{aligned}
$$

with

$$
\gamma_{1}=\left(\beta_{e} e \partial_{e}+\beta_{m_{H}} m_{H} \partial_{m_{H}}-2 \beta_{e} \xi \partial_{\xi}\right) \ln z=\mathcal{O}\left(\hbar^{2}\right)
$$




$$
\begin{aligned}
\hat{\gamma}_{s} & =2 \beta_{e}-\gamma_{s}-\gamma_{1} \\
\alpha_{1} & =\left(\gamma_{1}+\gamma_{s}-\beta_{e}\right) \hat{\xi}_{A}+\bar{\gamma}_{s} \xi_{A}+\left(\beta_{e} e \partial_{e}+\beta_{m_{H}} m_{H} \partial_{m_{H}}-2 \beta_{e} \xi \partial_{\xi}\right) \hat{\xi}_{A} \\
\hat{\alpha}_{1} & =\left(\gamma_{1}+\hat{\gamma}_{s}-\beta_{e}\right) \xi_{A}
\end{aligned}
$$

In (9.16), (9.17) we have already incorporated the relations (9.14) and (9.15). Therefore only the coefficient functions $\beta_{e}, \beta_{m_{H}}, \gamma_{s}, \bar{\gamma}_{s}$ and the coefficient $\alpha_{i n v}=\frac{1}{2} m_{H}^{2}+\mathcal{O}(\hbar)$ of the soft insertion $\int \delta_{\hat{\varphi}_{0}}$ turn out to be independent and have to be determined by explicit calculations (see [9]).

\subsection{Dependence on the ghost mass}

Due to the normalization condition (2.17) the dependence of the theory on the ghost mass is encoded in the differential operator $\xi_{A} \partial_{\xi_{A}}$ and we have to analyse the r.h.s. of $\xi_{A} \partial_{\xi_{A}} \Gamma=$ ?. This analysis almost completely parallels the analysis of the CS equation, but with one minor change: Instead of (8.2) we now have:

$$
0=\xi_{A} \partial_{\xi_{A}} \mathcal{S}(\Gamma)=s_{\Gamma}\left(\xi_{A} \partial_{\xi_{A}} \Gamma\right)-\chi_{A} \partial_{\xi_{A}} \Gamma
$$

However, differentiating the ST identity with respect to $\chi_{A}$ we find

$$
s_{\Gamma}\left(\partial_{\chi_{A}} \Gamma\right)=\partial_{\xi_{A}} \Gamma
$$

Hence the action principle, (9.18) and (9.19) imply that

$$
\left(\xi_{A} \partial_{\xi_{A}}+\chi_{A} \partial_{\chi_{A}}\right) \Gamma=\Delta_{\xi_{A}} \cdot \Gamma
$$

where $\Delta_{\xi_{A}} \cdot \Gamma$ is a BRS symmetric insertion. But due to

$$
s_{\Gamma}\left(\xi_{A} \partial_{\chi_{A}} \Gamma\right)=\left(s_{\Gamma} \xi_{A}\right) \partial_{\chi_{A}} \Gamma+\xi_{A} s_{\Gamma}\left(\partial_{\chi_{A}} \Gamma\right)=\chi_{A} \partial_{\chi_{A}} \Gamma+\xi_{A} \partial_{\xi_{A}} \Gamma
$$

$\Delta_{\xi_{A}} \cdot \Gamma$ also has to be a BRS variation and hence only BRS variations can contribute to $\Delta_{\xi_{A}} \cdot \Gamma$. From here on the discussion is completely analogous to the discussion of the CS equation; we skip the details and just present the result:

$$
\begin{gathered}
\left(\xi_{A} \tilde{\partial}_{\xi_{A}}+\chi_{A} \partial_{\chi_{A}}+\tilde{\beta}_{\xi}^{\xi_{A}} \tilde{\partial}_{\xi}-\tilde{\gamma}_{s}^{\xi_{A}} \mathcal{N}_{s}^{\left(\chi, \chi_{A}\right)}-\tilde{\hat{\gamma}}_{s}^{\xi_{A}} \hat{\mathcal{N}}_{s}^{\left(\chi, \chi_{A}\right)}-\tilde{\bar{\gamma}}_{s}^{\xi_{A}} \overline{\mathcal{N}}_{s}^{\left(\chi, \chi_{A}\right)}-\tilde{\gamma}_{B}^{\xi_{A}} \mathcal{N}_{B}^{\left(\chi, \chi_{A}\right)}\right) \Gamma \\
=\left(\chi \partial_{\xi}+\chi_{A} \partial_{\xi_{A}}\right) z^{-1} \xi_{A} \partial_{\xi_{A}} \int z Y_{1}\left(\varphi_{1}-\hat{\xi}_{A} \frac{m}{e}\right)
\end{gathered}
$$

In (9.22) $\xi_{A} \tilde{\partial}_{\xi_{A}}$ is the $W^{g e n}$-symmetric extension of $\xi_{A} \partial_{\xi_{A}}$ and given by:

$$
\begin{aligned}
\xi_{A} \tilde{\partial}_{\xi_{A}} \equiv & \xi_{A} \partial_{\xi_{A}}-z^{-1} \xi_{A} \partial_{\xi_{A}} \int z\left\{\left(\varphi_{1}-\hat{\xi}_{A} \frac{m}{e}\right) \frac{\delta}{\delta \varphi_{1}}+\left(\hat{\varphi}_{1}-\xi_{A} \frac{m}{e}\right) \frac{\delta}{\delta \hat{\varphi}_{1}}-Y_{1} \frac{\delta}{\delta Y_{1}}+q_{1} \frac{\delta}{\delta q_{1}}\right\} \\
& -\left(\chi \partial_{\xi}+\chi_{A} \partial_{\xi_{A}}\right) z^{-1} \xi_{A} \partial_{\xi_{A}} \int z\left(\hat{\varphi}_{1}-\xi_{A} \frac{m}{e}\right) \frac{\delta}{\delta q_{1}}
\end{aligned}
$$


Introducing the "real" $\beta$ - and $\gamma$-functions like in (9.13) the test of (9.22) on the gauge condition (4.2) yields:

$$
\begin{aligned}
\gamma_{B}^{\xi_{A}} & =0 \\
\beta_{\xi}^{\xi_{A}}=2 \gamma_{B}^{\xi_{A}} & =0 \\
-\gamma_{s}^{\xi_{A}}-\hat{\gamma}_{s}^{\xi_{A}}-\gamma_{B}^{\xi_{A}}=\left(\xi_{A} \partial_{\xi_{A}}+\beta_{\xi}^{\xi_{A}} \xi \partial_{\xi}\right) \ln z & \Leftrightarrow \hat{\gamma}_{s}^{\xi_{A}}=-\gamma_{s}^{\xi_{A}}-\xi_{A} \partial_{\xi_{A}} \ln z
\end{aligned}
$$

Again, we can separate in (9.22) the hard and soft breaking on the left and right hand side; thereby using (9.24) we end up with the following form, which for brevity we only give for all external fields set equal to zero:

$$
\begin{aligned}
& \left.\left(\xi_{A} \partial_{\xi_{A}}+\chi_{A} \partial_{\chi_{A}}-\gamma_{s}^{\xi_{A}} N_{s}-\hat{\gamma}_{s}^{\xi_{A}} \hat{N}_{s}-\bar{\gamma}_{s}^{\xi_{A}} \bar{N}_{s}-\xi_{A} \partial_{\xi_{A}} \ln z \int \varphi_{1} \frac{\delta}{\delta \varphi_{1}}\right) \Gamma\right|_{\text {ext.f. } \equiv 0} \\
= & -\left.\frac{m}{e} \int\left\{\alpha \frac{\delta}{\delta \varphi_{1}}+\xi_{A}\left(1-\gamma_{s}^{\xi_{A}}\right) \frac{\delta}{\delta \hat{\varphi}_{1}}+\left(\chi \partial_{\xi}+\chi_{A} \partial_{\xi_{A}}\right) \xi_{A}\left(1-\gamma_{s}^{\xi_{A}}\right) \frac{\delta}{\delta q_{1}}\right\} \Gamma\right|_{\text {ext.f. } \equiv 0}
\end{aligned}
$$

with

$$
\alpha=-\hat{\gamma}_{s}^{\xi_{A}} \hat{\xi}_{A}+\bar{\gamma}_{s}^{\xi_{A}} \xi_{A}+\xi_{A} \partial_{\xi_{A}} \hat{\xi}_{A}=x \xi_{A}+\mathcal{O}(\hbar)
$$

\subsection{RG equation}

The derivation of the RG equation once more starts with the action principle

$$
\kappa \partial_{\kappa} \Gamma=\Delta_{\kappa} \cdot \Gamma
$$

which tells us that $\Delta_{\kappa} \cdot \Gamma$ has to be an insertion of dimension less than or equal to four, invariant under charge conjugation and in addition BRS symmetric due to (8.2). In order to arrive at a more convenient form of the RG equation we now introduce a new set of BRS symmetric operators (see also [10]) representing the two- and three-dimensional BRS symmetric classical field polynomials, i.e. instead of (8.19) and $\int \delta_{\hat{\varphi}_{0}}$ we are going to use:

$$
\begin{array}{ll}
m \partial_{m} & , \quad f_{A} \partial_{\xi_{A}} \Gamma+\left[\left(\chi \partial_{\xi}+\chi_{A} \partial_{\xi_{A}}\right) f_{A}\right] \partial_{\chi_{A}} \Gamma=s_{\Gamma}\left(f_{A} \partial_{\chi_{A}} \Gamma\right) \\
\int \frac{\delta}{\delta \hat{\varphi}_{0}} & , \quad \tilde{f} \int\left\{B \hat{\varphi}_{2}-\bar{c} q_{2}\right\}+\left(\chi \partial_{\xi}+\chi_{A} \partial_{\xi_{A}}\right) \tilde{f} \int \bar{c} \hat{\varphi}_{2}=s_{\Gamma}\left(\tilde{f} \int \bar{c} \hat{\varphi}_{2}\right)
\end{array}
$$

Hence according to BRS invariance alone, $\Delta_{\kappa} \cdot \Gamma$ can be decomposed into a sum of the BRS symmetric operators (9.28), (8.20) and (the remaining four-dimensional operators in) (8.17):

$$
\begin{aligned}
\kappa \partial_{\kappa} \Gamma= & \left(-\beta_{m}^{\kappa} m \partial_{m}-\tilde{\beta}_{\xi_{A}}^{\kappa}\left(f_{A} \partial_{\xi_{A}}+\left[\left(\chi \partial_{\xi}+\chi_{A} \partial_{\xi_{A}}\right) f_{A}\right] \partial_{\chi_{A}}+\alpha_{i n v}^{\kappa} \int \delta_{\hat{\varphi}_{0}}-\beta_{m_{H}}^{\kappa} m_{H} \partial_{m_{H}}\right.\right. \\
& -\beta_{e}^{\kappa} e \partial_{e}+\gamma_{A}^{\kappa} N_{A}+\tilde{\gamma}_{B}^{\kappa} \mathcal{N}_{B}^{\left(\chi, \chi_{A}\right)}+\sum_{i=1}^{2}\left\{\tilde{\gamma}_{s, i}^{\kappa} \mathcal{N}_{s, i}^{\left(\chi, \chi_{A}\right)}+\tilde{\tilde{\gamma}}_{s, i}^{\kappa} \overline{\mathcal{N}}_{s, i}^{\left(\chi, \chi_{A}\right)}+\tilde{\hat{\gamma}}_{s, i}^{\kappa} \hat{\mathcal{N}}_{s, i}^{\left(\chi, \chi_{A}\right)}\right\} \\
& -\tilde{\beta}_{\xi}^{\kappa}\left(f_{\xi} \partial_{\xi}+\left[\left(\chi \partial_{\xi}+\chi_{A} \partial_{\xi_{A}}\right) f_{\xi}\right] \partial_{\chi}\right) \Gamma+\tilde{\gamma}^{\kappa} s_{\Gamma}\left(\tilde{f}_{3} \int \bar{c} \hat{\varphi}_{2}\right)+\tilde{\tilde{\gamma}}^{\kappa} s_{\Gamma}\left(\tilde{f}_{4} \int \bar{c} \hat{\varphi}_{1} \hat{\varphi}_{2}\right)(9.29)
\end{aligned}
$$


Differentiating (9.29) with respect to $\varphi_{1}$, setting all fields equal to zero and making use of the normalization condition $\Gamma_{\varphi_{1}}=0(2.20)$ it immeadiately follows that:

$$
\alpha_{i n v}^{\kappa} \equiv 0
$$

With this result in mind three further tests of (9.29) on the physical normalization conditions (2.17) concerning the mass normalizations of the Higgs, the vector and the ghost directly imply

$$
\beta_{m_{H}}^{\kappa} \equiv 0 \quad, \quad \beta_{m}^{\kappa} \equiv 0 \quad, \quad \tilde{\beta}_{\xi_{A}}^{\kappa} \equiv 0
$$

to all orders of perturbation theory. Therefore due to the physical normalization conditions the first line of the r.h.s. of $(9.29)$ is absent and no $\beta$-function in connection with a (physical) mass appears in the RG equation.

In order to conclude the derivation of the $\mathrm{RG}$ equation we now have to exploit rigid invariance of the theory: To this end we first apply $W^{\text {gen }}$ (6.7) to the RG equation (9.29) and then also use the rigid WI (6.10):

$$
W^{g e n} \kappa \partial_{\kappa} \Gamma=\left[W^{g e n}, \kappa \partial_{\kappa}\right] \Gamma+\kappa \partial_{\kappa} W^{g e n} \Gamma=-\left(\kappa \partial_{\kappa} W^{g e n}\right) \Gamma+\kappa \partial_{\kappa}\left(\chi \Delta_{b r}+\chi_{A} \Delta_{b r_{A}}\right)
$$

This leads after some calculation to the final form of the RG equation:

$$
\begin{aligned}
& \left(\kappa \partial_{\kappa}+\beta_{e}^{\kappa} e \partial_{e}+\beta_{\xi}^{\kappa} \xi \partial_{\xi}-\gamma_{A}^{\kappa} N_{A}-\gamma_{B}^{\kappa} N_{B}-\gamma_{s}^{\kappa} N_{s}-\hat{\gamma}_{s}^{\kappa} \hat{N}_{s}-\bar{\gamma}_{s}^{\kappa} \bar{N}_{s}\right. \\
& -\gamma_{1}^{\kappa} \int\left\{\varphi_{1} \frac{\delta}{\delta \varphi_{1}}-Y_{1} \frac{\delta}{\delta Y_{1}}+\hat{\varphi}_{1} \frac{\delta}{\delta \hat{\varphi}_{1}}+q_{1} \frac{\delta}{\delta q_{1}}\right\} \\
& \left.-\left(\chi \partial_{\xi}+\chi_{A} \partial_{\xi_{A}}\right)\left[\int\left\{\left(\hat{\gamma}_{s}^{\kappa}+\gamma_{1}^{\kappa}\right) \hat{\varphi}_{1} \frac{\delta}{\delta q_{1}}+\hat{\gamma}_{s}^{\kappa} \hat{\varphi}_{2} \frac{\delta}{\delta q_{2}}+\gamma_{B}^{\kappa} \bar{c} \frac{\delta}{\delta B}\right\}-\beta_{\xi}^{\kappa} \xi \partial_{\chi}\right]\right) \Gamma \\
= & \bar{\gamma}_{s}^{\kappa} \int\left\{q_{1} Y_{1}+q_{2} Y_{2}\right\} \\
& +\left(\chi \partial_{\xi}+\chi_{A} \partial_{\xi_{A}}\right) \int\left\{\left(\gamma_{s}^{\kappa}+\gamma_{1}^{\kappa}\right) Y_{1} \varphi_{1}+\gamma_{s}^{\kappa} Y_{2} \varphi_{2}+\bar{\gamma}_{s}^{\kappa}\left(Y_{1} \hat{\varphi}_{1}+Y_{2} \hat{\varphi}_{2}\right)\right\}
\end{aligned}
$$

with

$$
\gamma_{1}^{\kappa}=\left(\kappa \partial_{\kappa}+\beta_{e}^{\kappa} e \partial_{e}+\beta_{\xi}^{\kappa} \xi \partial_{\xi}\right) \ln z
$$

In (9.33) we have already introduced the full $\beta$ - and $\gamma$-functions of the RG equation like in (9.13). Again, our analysis shows that the $\beta$-function $\beta_{e}^{\kappa}$ and the anomalous dimension $\gamma_{A}^{\kappa}$ have to be $\xi$ - and $\xi_{A}$-independent to all orders of the loop expansion.

Additionally, rigid invariance (9.32) also imposes two restrictions for the coefficient functions of the RG equation:

$$
\begin{aligned}
\left(\kappa \partial_{\kappa}+\beta_{\xi}^{\kappa} \xi \partial_{\xi}+\beta_{e}^{\kappa} e \partial_{e}\right)\left(z \hat{\xi}_{A} \frac{m}{e}\right) & =-z \xi_{A} \frac{m}{e} \bar{\gamma}_{s}^{\kappa}-z \hat{\xi}_{A} \frac{m}{e} \gamma_{s}^{\kappa} \\
\beta_{e}^{\kappa}-\hat{\gamma}_{s}^{\kappa} & =\left(\kappa \partial_{\kappa}+\beta_{\xi}^{\kappa} \xi \partial_{\xi}+\beta_{e}^{\kappa} e \partial_{e}\right) \ln z
\end{aligned}
$$


Some further information about the coefficient functions results from testing the RG equation on the gauge condition (4.2)):

$$
\begin{gathered}
\gamma_{B}^{\kappa}=-\gamma_{A}^{\kappa} \\
\beta_{\xi}^{\kappa}=2 \gamma_{B}^{\kappa}=-2 \gamma_{A}^{\kappa} \\
\gamma_{s}^{\kappa}=-\gamma_{B}^{\kappa}=\gamma_{A}^{\kappa}
\end{gathered}
$$

Hence also $\gamma_{B}^{\kappa}, \gamma_{s}^{\kappa}$ and $\beta_{\xi}^{\kappa}$ are fully gauge parameter independent.

Finally, one further relation emerges from the validity of the local WI (7.14) and the normalization condition for the coupling (7.16):

$$
\gamma_{A}^{\kappa}=\beta_{e}^{\kappa}
$$

Therefore, there is only one independent coefficient function appearing in the RG equation, namely the $\beta$-function $\beta_{e}^{\kappa}$, which has to be determined by an explicit calculation.

\section{Conclusions}

In the present paper we have examined the renormalization of the Abelian Higgs model including BRS variations of all the gauge parameters. The advantage of such an extended procedure (when compared to the usual one) is due to the fact that this procedure also yields full information about the gauge parameter dependence of 1-PI Green functions automatically and in an easily manageable way and therefore prohibits (just by construction) a wrong adjustment of counterterms which in turn would spoil the gauge parameter independence of the S-matrix. In the usual construction (i.e. without introducing BRS transforming gauge parameters) such a simple guiding principle is missing and it is a quite troublesome and heavily controllable task to adjust the counterterms correctly.

In this context we have shown that the normalization conditions needed in order to fix the free parameters of the theory cannot be chosen arbitrarily but instead have to respect the restrictions dictated by the enlarged ST identity. Especially we have proven that the physical on-shell normalization conditions are in complete agreement with those restrictions. Furthermore, the method of BRS varying gauge parameters yields a well handleable tool for controlling the range of "good" normalization conditions, i.e. normalization conditions, which are not in contradiction with the enlarged ST identity.

Some further results of the algebraic method we find interesting, too:

\footnotetext{
${ }^{7}$ When deriving (9.36) we make use of (9.35).
} 
The enlarged ST identity also allowed us to show that the transversal part of the vector 2-point function has to be completely gauge parameter-independent to all orders of perturbation theory.

In the course of proving the local WI we found the $\xi$-and $\xi_{A}$-independence of the overall normalization factor of the matter transformations, a result, which gave rise to an alternative and elegant possibility for fixing the coupling, namely by requiring the local WI to be exact to all orders.

Finally, we derived the Callan-Symanzik and the renormalization group equation of the Abelian Higgs model thereby showing among other things that the $\beta$-functions $\beta_{e}^{(\kappa)}, \beta_{m_{H}}$ and $\beta_{\xi}^{(\kappa)}$ as well as the anomalous dimensions $\gamma_{A}^{(\kappa)}, \gamma_{B}^{(\kappa)}$ and $\gamma_{s}^{\kappa}$ have to be fully gauge parameter-independent to all orders of the perturbative expansion.

The examination of the Abelian Higgs model, chosen as the simplest example of a gauge theory with spontaneous breakdown of symmetry, thus clearly shows of what kind the considerations have to be and yields a hint what kind of results could possibly be expecxted when the general algebraic method will be applied to more complicated, physical, models, especially to the standard model of electroweak interactions.

Acknowledgements The authors would like to thank K. Sibold and E. Kraus for numerous helpful discussions and a critical reading of the manuscript.

\section{Appendix A}

In the course of looking for the most general classical solution of the $\chi$ - and $\chi_{A}$-enlarged ST identity (4.1) the most general solution of the gauge condition (4.2) and the ordinary (that is $\chi$ - and $\chi_{A}$-independent) ST identity

$$
\mathcal{S}(\hat{\Gamma})=\int\left\{\partial_{\mu} c \frac{\delta \hat{\Gamma}}{\delta A_{\mu}}+B \frac{\delta \hat{\Gamma}}{\delta \bar{c}}+\frac{\delta \hat{\Gamma}}{\delta \underline{Y}} \frac{\delta \hat{\Gamma}}{\delta \underline{\varphi}}+\underline{q} \frac{\delta \hat{\Gamma}}{\delta \underline{\hat{\varphi}}}\right\}=0
$$

is needed. This solution was constructed in [9], and we just present the result here:

$$
\hat{\Gamma}_{c l}^{g e n}=\Lambda\left(A_{\mu}, \bar{\varphi}_{1}, \bar{\varphi}_{2}\right)+\Gamma_{g . f .}+\Gamma_{\phi \pi}+\Gamma_{e . f .},
$$

with

$$
\bar{\varphi}_{i}=\varphi_{i}-x_{i} \hat{\varphi}_{i}, i=1,2 .
$$

The part $\Lambda=\Lambda\left(A_{\mu}, \bar{\varphi}_{1}, \bar{\varphi}_{2}\right)$ describing the gauge field $A_{\mu}$ and matter fields $\varphi_{i}$ is given by:

$$
\Lambda=\int\left\{-\frac{z_{A}}{4} F_{\mu \nu} F^{\mu \nu}+\frac{1}{2} z_{1}\left(\partial_{\mu} \bar{\varphi}_{1}\right)\left(\partial^{\mu} \bar{\varphi}_{1}\right)+\frac{1}{2} z_{2}\left(\partial_{\mu} \bar{\varphi}_{2}\right)\left(\partial^{\mu} \bar{\varphi}_{2}\right)\right.
$$




$$
\begin{aligned}
& +z_{e} e \sqrt{z_{1}} \sqrt{z_{2}} \sqrt{z_{A}}\left(\left(\partial_{\mu} \bar{\varphi}_{1}\right) \bar{\varphi}_{2}-\bar{\varphi}_{1}\left(\partial_{\mu} \bar{\varphi}_{2}\right)\right) A^{\mu}+\frac{1}{2} z_{e}^{2} e^{2} z_{A}\left(z_{1} \bar{\varphi}_{1}^{2}+z_{2} \bar{\varphi}_{2}^{2}\right) A_{\mu} A^{\mu} \\
& +\frac{1}{2} z_{m} m^{2} z_{A} A_{\mu} A^{\mu}-\sqrt{z_{2}} \sqrt{z_{m}} m \sqrt{z_{A}}\left(\partial_{\mu} \bar{\varphi}_{2}\right) A^{\mu}+z_{e} e \sqrt{z_{m}} m \sqrt{z_{1}} z_{A} \bar{\varphi}_{1} A_{\mu} A^{\mu} \\
& +\frac{1}{2} \mu^{2}\left(z_{1} \bar{\varphi}_{1}^{2}+2 \sqrt{z_{1}} \frac{\sqrt{z_{m}} m}{z_{e} e} \bar{\varphi}_{1}+z_{2} \bar{\varphi}_{2}^{2}\right) \\
& \left.-\frac{1}{8} \frac{z_{m_{H}} m_{H}^{2}}{z_{m} m^{2}} z_{e}^{2} e^{2}\left(z_{1} \bar{\varphi}_{1}^{2}+2 \sqrt{z_{1}} \frac{\sqrt{z_{m}} m}{z_{e} e} \bar{\varphi}_{1}+z_{2} \bar{\varphi}_{2}^{2}\right)^{2}\right\}
\end{aligned}
$$

The gauge fixing part $\Gamma_{\text {g.f. }}$ immediately results from integrating the gauge condition (4.2):

$$
\Gamma_{g . f .}=\int\left\{\frac{1}{2} \xi B^{2}+B \partial A-e B\left[\left(\hat{\varphi}_{1}-\xi_{A} \frac{m}{e}\right) \varphi_{2}-\hat{\varphi}_{2}\left(\varphi_{1}-\hat{\xi}_{A} \frac{m}{e}\right)\right]\right\}
$$

For the remaining two parts, the external field part $\Gamma_{e . f}$. and the $\phi \pi$-part $\Gamma_{\phi \pi}$, one gets

$$
\Gamma_{e . f .}=\int\left\{Y_{1}\left(-e z_{e} \sqrt{\frac{z_{2}}{z_{1}}} \sqrt{z_{A}} \bar{\varphi}_{2} c+x_{1} q_{1}\right)+Y_{2}\left(e z_{e} \sqrt{\frac{z_{1}}{z_{2}}} \sqrt{z_{A}}\left(\bar{\varphi}_{1}+\frac{\sqrt{z_{m}} m}{\sqrt{z_{1}} z_{e} e}\right) c+x_{2} q_{2}\right)\right\}
$$

and

$$
\begin{aligned}
\Gamma_{\phi \pi}= & \int\left\{-\bar{c} \square c+e \bar{c}\left(q_{1} \varphi_{2}-q_{2}\left(\varphi_{1}-\hat{\xi}_{A} \frac{m}{e}\right)\right)\right. \\
& +e \bar{c}\left(\hat{\varphi}_{1}-\xi_{A} \frac{m}{e}\right)\left(z_{e} e \sqrt{\frac{z_{1}}{z_{2}}} \sqrt{z_{A}}\left(\bar{\varphi}_{1}+\frac{\sqrt{z_{m}} m}{\sqrt{z_{1}} z_{e} e}\right) c+x_{2} q_{2}\right) \\
& \left.-e \bar{c} \hat{\varphi}_{2}\left(-z_{e} e \sqrt{\frac{z_{2}}{z_{1}}} \sqrt{z_{A}} \bar{\varphi}_{2} c+x_{1} q_{1}\right)\right\} .
\end{aligned}
$$

The free parameters in the general solution of the ST identity (A.1) are the wave function normalizations $z_{1}, z_{2}$ and $z_{A}$, the mass renormalizations of the vector and the Higgsparticle, i.e. $z_{m}, z_{m_{H}}$, the coupling renormalization $z_{e}$, the parameters $x_{1}, x_{2}$, the parameter $\mu$, the gauge parameters $\xi, \xi_{A}$ and the parameter $\hat{\xi}_{A}$. These parameters are not prescribed by the ST identity (A.1) and therefore have to be fixed by appropriate normalization conditions to all orders (see section 2).

\section{Appendix B}

The solution of (8.15) was given in [9]; first we present a list of all terms of dimension less than or equal to three which contribute to $\hat{\Delta}_{\lambda}^{0}$ :

$$
\begin{aligned}
\int \frac{\hat{\Gamma}_{c l}}{\delta \varphi_{1}} & =s_{\hat{\Gamma}_{c l}^{\chi=0=\chi_{A}}} \int Y_{1}, \\
\int \frac{\delta \hat{\Gamma}_{c l}}{\delta \hat{\varphi}_{1}} & =s_{\hat{\Gamma}_{c l}^{\chi=0=\chi_{A}}}^{\chi} \int\left\{-x Y_{1}-e \bar{c} \bar{\varphi}_{2}\right\}, \\
\int\left\{B \hat{\varphi}_{2}-\bar{c} q_{2}\right\} & =s_{\hat{\Gamma}_{c l}^{\chi=0=\chi_{A}}}^{\chi} \int \bar{c} \hat{\varphi}_{2}
\end{aligned}
$$


and

$$
\int\left\{z_{1} \bar{\varphi}_{1}^{2}+2 z_{1} v \bar{\varphi}_{1}+z_{2} \bar{\varphi}_{2}^{2}\right\}
$$

In order to have a proper definition of this last invariant in higher orders we are forced to introduce a further external field $\hat{\varphi}_{0}$ of dimension two, even under charge conjugation and invariant under BRS and rigid transformations, which couples to this invariant. Therefore the above BRS symmetric term is replaced by:

$$
\int \frac{\delta \hat{\Gamma}_{c l}}{\delta \hat{\varphi}_{0}}
$$

The four-dimensional BRS symmetric terms contributing to $\hat{\Delta}_{\lambda}^{0}$ are given by $(i=1,2)$ :

$$
\begin{aligned}
& \int\left\{\varphi_{i} \frac{\delta}{\delta \varphi_{i}}-Y_{i} \frac{\delta}{\delta Y_{i}}\right\} \hat{\Gamma}_{c l}=s_{\hat{\Gamma}_{c l}=0=\chi_{A}}^{\chi} \int Y_{i} \varphi_{i}, \\
& \int\left\{\hat{\varphi}_{i} \frac{\delta \hat{\Gamma}_{c l}}{\delta \varphi_{i}}-Y_{i} q_{i}\right\}=s_{\hat{\Gamma}_{c l}}^{\chi=0=\chi_{A}} \int Y_{i} \hat{\varphi}_{i}, \\
& \int\left\{B \frac{\delta}{\delta B}+\bar{c} \frac{\delta}{\delta \bar{c}}\right\} \hat{\Gamma}_{c l}=s_{\hat{\Gamma}_{c l}}^{\chi=0=\chi_{A}} \int \bar{c} \frac{\delta \hat{\Gamma}_{c l}}{\delta B}, \\
& \int\left\{\hat{\varphi}_{i} \frac{\delta}{\delta \hat{\varphi}_{i}}+q_{i} \frac{\delta}{\delta q_{i}}\right\} \hat{\Gamma}_{c l}=s_{\hat{\Gamma}_{c l}=0=\chi_{A}}^{\chi=} \hat{\varphi}_{i} \frac{\delta \hat{\Gamma}_{c l}}{\delta q_{i}}, \\
& \int\left\{B \hat{\varphi}_{1} \hat{\varphi}_{2}-\bar{c} q_{1} \hat{\varphi}_{2}-\bar{c} \hat{\varphi}_{1} q_{2}\right\}=s_{\hat{\Gamma}_{c l}=0=\chi_{A}}^{\chi} \int \bar{c} \hat{\varphi}_{1} \hat{\varphi}_{2}, \\
& \xi \partial_{\xi} \hat{\Gamma}_{c l}=s_{\hat{\Gamma}_{c l}}^{\chi=0=\chi_{A}} \xi Q
\end{aligned}
$$

and

$$
\int\left\{A \frac{\delta}{\delta A}+c \frac{\delta}{\delta c}\right\} \hat{\Gamma}_{c l} \quad, \quad m_{H} \partial_{m_{H}} \hat{\Gamma}_{c l} \quad, \quad e \partial_{e} \hat{\Gamma}_{c l}
$$

Please note that due to (B.14) the coefficients with which the terms in (B.2) and (B.4) appear in $\hat{\Delta}_{\lambda}^{0}$ are independent of both $\xi$ and $\xi_{A}$.

\section{Appendix C}

In this appendix we present the $W^{g e n}$-symmetric extensions of the BRS invariant insertions

$$
m_{H} \partial_{m_{H}}, \quad e \partial_{e}, \quad f_{\xi} \partial_{\xi}+\left(\chi \partial_{\xi}+\chi_{A} \partial_{\xi_{A}}\right) f_{\xi} \partial_{\chi} .
$$

Just in order to compactify the notation in the formulae below we introduce two $\xi$ - and $\xi_{A^{-}}$ independent factors $f_{H}$ and $f_{e}$ multiplying $m_{H} \partial_{m_{H}}$ and $e \partial_{e}$, respectively. (These factors have to be independent of $\xi$ and $\xi_{A}$ due to the results of section 8.) Next we define 
$(i=H, e, \xi)$ :

$$
\begin{aligned}
\nabla_{i}= & m_{H} \partial_{m_{H}}, e \partial_{e}, \partial_{\xi} \\
\hat{\nabla}_{i}= & -f_{i} \frac{1}{z} \nabla_{i} \int z\left\{\left(\varphi_{1}-\hat{\xi}_{A} \frac{m}{e}\right) \frac{\delta}{\delta \varphi_{1}}-Y_{1} \frac{\delta}{\delta Y_{1}}+\left(\hat{\varphi}_{1}-\xi_{A} \frac{m}{e}\right) \frac{\delta}{\delta \hat{\varphi}_{1}}+q_{1} \frac{\delta}{\delta q_{1}}\right\} \\
& -\left(\chi \partial_{\xi}+\chi_{A} \partial_{\xi_{A}}\right) f_{i} \frac{1}{z} \nabla_{i} \int z\left(\hat{\varphi}_{1}-\xi_{A} \frac{m}{e}\right) \frac{\delta}{\delta q_{1}} \\
\hat{\vec{\nabla}}_{i} \Gamma= & -\left(\chi \partial_{\xi}+\chi_{A} \partial_{\xi_{A}}\right) f_{i} \frac{1}{z} \nabla_{i} \int z Y_{1}\left(\varphi_{1}-\hat{\xi}_{A} \frac{m}{e}\right)
\end{aligned}
$$

The $W^{g e n}$-symmetric extensions of the operators in (C.1) then are given by:

$$
\begin{aligned}
f_{H} m_{H} \partial_{m_{H}} \Gamma & \rightarrow f_{H} m_{H} \tilde{\partial}_{m_{H}} \Gamma \equiv f_{H} m_{H} \partial_{m_{H}} \Gamma+\hat{\nabla}_{H} \Gamma+\hat{\hat{\nabla}}_{H} \Gamma \\
f_{e} e \partial_{e} \Gamma & \rightarrow f_{e} e \tilde{\partial}_{e} \Gamma \equiv f_{e} e \partial_{e} \Gamma+\hat{\nabla}_{e} \Gamma+\hat{\nabla}_{e} \Gamma \\
f_{\xi} \partial_{\xi} \Gamma+\left[\left(\chi \partial_{\xi}+\chi_{A} \partial_{\xi_{A}}\right) f_{\xi}\right] \partial_{\chi} \Gamma & \rightarrow \tilde{\partial}_{\xi} \Gamma \equiv f_{\xi} \partial_{\xi} \Gamma+\left[\left(\chi \partial_{\xi}+\chi_{A} \partial_{\xi_{A}}\right) f_{\xi}\right] \partial_{\chi} \Gamma+\hat{\nabla}_{\xi} \Gamma+\hat{\hat{\nabla}}_{\xi} \Gamma
\end{aligned}
$$




\section{References}

[1] B.W. Lee and J. Zinn-Justin, Phys. Rev. D7 (1973) 1049.

[2] C. Becchi, A. Rouet and R. Stora, Ann. Phys. (NY) 98 (1976) 287.

[3] H. Kluberg-Stern and J.B. Zuber, Phys. Rev. D12 (1975) 467, 482.

[4] O. Piguet and K. Sibold, Nucl. Phys. B253 (1985) 517.

[5] R. Häußling and E. Kraus, Z. Phys. C 75 (1997).

[6] N.K. Nielsen, Nucl. Phys. B101 (1975) 173.

[7] E. Kraus, Habilitationsschrift, Bonn (1997).

[8] E. Kraus and K. Sibold, Nucl. Phys. 331 (1990) 350.

[9] E. Kraus and K. Sibold, Z. Phys. C 68 (1995) 331.

[10] E. Kraus, Z. Phys. C 60 (1993) 741. 